\title{
An array multi-physics acquisition system with focus on reser- voir monitoring
}

\author{
K. Strack ${ }^{1}$, S. Davydycheva ${ }^{1}$, T. Hanstein ${ }^{1}$, A. Y. Paembonan ${ }^{1,2}$ and M. Smirnov ${ }^{1,3}$ \\ 1 KMS Technologies - KJT Enterprises Inc. - Houston, Texas USA \\ 2 Department of Geophysical Engineering, Sumatera Institute of Technology, Indonesia \\ 3 Department of Civil, Environmental and Natural Resources Engineering, Lulea University of Technology, \\ Sweden \\ * Correspondence: kur@@kmstechnologies.com (K.M.S); Tilman@kmstechnologies.com (T.H.)
}

\begin{abstract}
Focusing geophysics to improve recovery factor of hydrocarbon reservoirs adds value and contributes toward ZERO carbon footprint by increasing the recovery factor by of $30-40 \%$ and thus reducing the cost/carbon emission per produced barrel. Thus, the Enhanced Oil Recovery (EOR) market is expected to grow more than 3.5\% annually. This will be even more fueled by the GreenHouse-Gas (GHG) reduction and subsequent $\mathrm{CO}_{2}$ injection into the reservoirs as they are being produced. Presently, geophysics only accounts for a small percentage of this market, thus its growth is inevitable since more deterministic observation lead higher operating efficiency. Imaging the fluids (hydrocarbon, water, and $\mathrm{CO}_{2}$ ) is a key component to optimized production and injection.We designed a novel electromagnetic (EM) acquisition system that combines multi-physics fluid imaging and acquires surface and borehole data with high fidelity. Borehole calibration is needed to upscale reservoir data and parameters to measurement scale. Multiple electromagnetic methods are used as well as microseismics in one layout for Exploration and Production (E \& P) problems. Multi-components in electromagnetics allows resolving oil and water-bearing zones equally well while achieving the best accuracy suitable for repeat measurements. Because sedimentary basins are intrinsically anisotropic, considering 3-dimensional anisotropy is essential from measurement and 3D modeling viewpoint. Thus, the results have the better subsurface images.Here, we combine hardware design, methodology, 3D modeling, processing, and interpretations into an integrated technology and demonstrate the success with verifiable case histories.
\end{abstract}

Keywords: Array electromagnetics; magnetotellurics; controlled source electromagnetics; microseismics; reservoir monitoring; enhanced oil recovery; recovery factor improvement

\section{Introduction}

Focusing on applied geophysics, with oil price fluctuations, to improve recovery factor of hydrocarbon reservoirs can add significant value and contributes towards the transition to zero carbon footprint. Enhanced Oil Recovery (EOR) improved the average recovery factor for typical mature oil fields worldwide average by about $35 \%$ [1]. As a result, it has been gained more than 50 years of oil supply. The EOR market alone was over 20 billion US \$ in 2015, with projected growth of more than $3.5 \%$ over the period of 20222027 (grandviewresearch.com, market study report). Geophysics only accounts for a small percentage of this market, thus growth in geophysical measurements is obvious since they can directly improve operating efficiency. For fluid imaging, electromagnetic (EM) is the most direct method compared to the other geophysical method. The appropriate EM methods were well developed in the 1980s and 1990s, but with the oil market downturn and better structural mapping capabilities of seismics, they were mostly limited to geothermal exploration. Since the start of using geophysics for hydrocarbon exploration, there were two historical periods of using EM methods in the industry (1950s and 1980s), before their application to marine exploration showed their commercial value [1]. Several review papers summarized the state of the technology and the industry [1, 3-12]. Now 
(2021), in the 3rd conceptual phase, we are still evaluating technology with respect to physics and geology (for industrial implementation). We had these technical limitations because we could not measure the electrical anisotropy in the borehole until 1999, when new logging tool appeared [35]. Recent publications by [13-15] show the commercial value and usage of the EM methods has reached an industrial level. EM reservoir monitoring has been investigated in several feasibility studies justifying fit-for-purpose acquisition systems. Here, we are mostly reviewing a land system, but the components can easily be translated to offshore applications.

EOR is always challenged by the knowledge of the oil-water (or steam) front location and flow direction. Only limited geophysical techniques have been applied to resolve this. Seal integrity is best addressed with microseismics [16] while water flood front best with EM [17-20]. Since the flooded reservoir is conductive and the hydrocarbon saturated part is resistive, it is necessary to have both magnetic and electric field data [17-18]. After careful 3D feasibility study and noise tests, we select Controlled Source Electromagnetics (CSEM) in the time domain as the most sensitive method for land/onshore applications $[17,19,20]$. From the 3D modeling, we derived as key requirement that borehole and surface data needed to be integrated by measuring between surface-to-borehole and calibrated using conventional logs including the resistivity anisotropy. This would significantly reduce the risk in interpretation [21-25].

Over the past 30 years magnetotellurics (MT) has been the 'work horse' in geothermal exploration and collaborated with other geophysical methods [3, 4]. Standard MT systems (operating at a frequency range from $0.0001 \mathrm{~Hz}$ to $1 \mathrm{kHz}$ ) and audio-magnetotelluric (AMT) systems (operating at a frequency range from $1 \mathrm{~Hz}$ to $20 \mathrm{kHz}$ ) are routinely used. While the methodology and technology has stabilized, they are limited by cost and by moderate number of data sites. Little innovation regarding operational and interpretational workflow and cost optimization [1] could be found. The main purpose of going to an array architecture is getting more data of better quality at lower cost and utilizing the strength of spatial data redundancy of as we can find in seismics.

MT is sufficient for most geothermal applications, where the target is more conductive than the background rock. For hydrocarbon applications MT is limited to general basin depth definition or sediment thickness imaging, since MT is mostly insensitive to thin horizontal resistors associated with hydrocarbon accumulations. CSEM with grounded electric dipole excitation is better suited for this, since the grounded transmitter excites both horizontal and vertical currents in the formation making the method sensitive to thin resistors and to the resistivity anisotropy [26].

For shale / unconventional application and reservoir monitoring, EM (mostly CSEM) response could yield more value than seismics in providing accurate information on the fluid properties. During the flooding operations, high-volume channels can gradually develop due to natural underground fractures, or they may occur suddenly, for example, with a hydraulic fracturing job [27]. EM monitoring methods, sometimes in combination with microseismic monitoring, can aid monitoring the reservoir seal during production. These combined methods deliver synergetic valuable information on the location and direction of the waterfront, due to the high sensitivity of the EM field to the fluid properties and the strong response of seismic to volumes boundaries (with impedance contrast).

Here, we will start with the biggest issue we need to address, anisotropy, because the error we make by ignoring it is between 30 to 50\%. After reviewing the technical advances, we derive the system requirement by keeping the reservoir monitoring (and therefore required time-laps measurements) in mind. Next are the specifications which are the translation of the requirements into something that we can build. The developed and fully field commercialized array system technology is then applied to various geologies in Europe, USA, and Asia.

\section{Importance of anisotropy}


The anisotropy is the single most important technical issues for real reservoirs and even more so for unconventional reservoirs as they often contain anisotropic clay minerals. The resistivity anisotropy is determined by the difference in electric rock properties across and along the layering. A moderate anisotropy is always present in shales, sands, and other sedimentary rocks due to layering during geologic deposition (see Figure 1) and additional anisotropy due to the shale content. In carbonates (most of the world's oil reservoirs), fluid filled fractured cause anisotropy in any direction. In shale-sand laminations the horizontal resistivity, $\mathrm{Rh}$, is typically low, being dominated by the conductive shales, whereas the vertical resistivity, Rv, may be high, dominated by the oil-saturated resistive sands. These reservoirs are often referred to as transverse isotropic reservoirs. Thus, their ratio $\mathrm{Rv} / \mathrm{Rh}$ can sometimes reach ten or even more. This can be seen in the logs in Figure 2 where the darker layers have higher shale content than the lighter layers with more sands. Since layering is intrinsic to the depositional environment, it can be found at every scale shown in the figure starting from electron microscope scale and going to reservoir scale. Electrical anisotropy significantly affects the CSEM measurements, so taking the anisotropy into account is critical. CSEM measurement correlate best with the vertical resistivity from tri-axial induction logs and MT measurements best with the horizontal resistivity from induction logs.

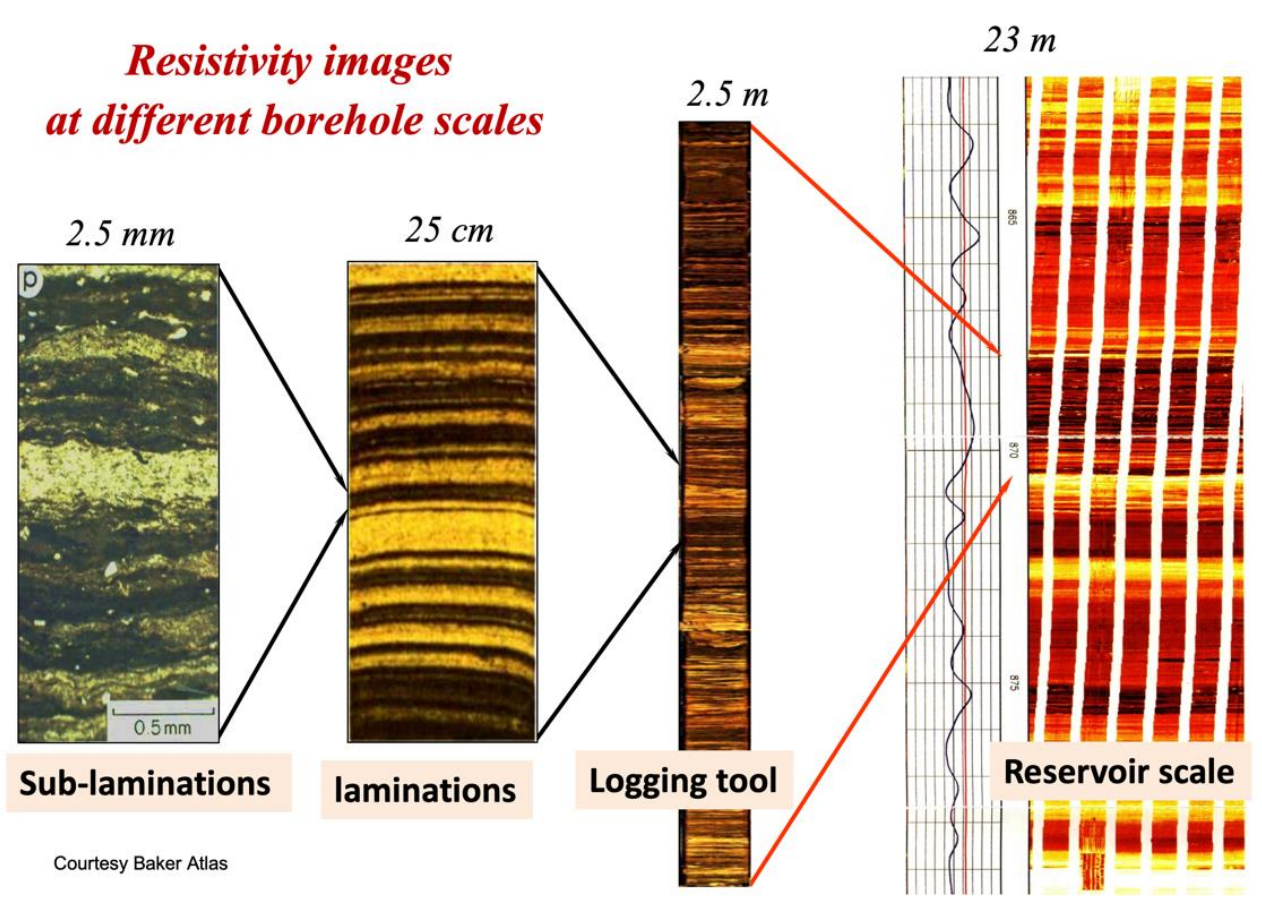

Figure 1. Examples of resistivity image logs at different scales showing the layering of sand-shale sequences at any scale. On the left is an electron microscope image at sub-lamination scale, then a core image at lamination scale ( 1 inch to 1 centimeter), this is followed by a resistivity image $\log (1$ track) from a logging tool, and to the right a typical electrical image log section from a 6-arm resistivity imager for a $20 \mathrm{~m}$ reservoir section (modified after [1]).

Shale formations have an inherent strong electrical anisotropy because of the clay minerals. A typical shale reservoir consists often of a sequence of relatively thin layers called laminations once they can be seen in borehole measurements. As the hydrocarbons in shale gas or shale oil reservoirs are mostly resistive and contained in the sand layers of the laminations, they also give an additional anomalous EM response. The Direct Hydrocarbon Indicator (DHI) effect also known as the 'thin resistive layer effect' [28,29] gave rise to the entire marine EM industry [30]. We are often surprised by the strong anomalous response of thin resistive layers at depth and the double effect of anisotropy and DHI 
maybe an explanation. We see this unusual anomalous response with the Bakken in North Dakota and have included it as example in this paper.

Over the past 20 years, new resistivity anisotropy logging tools have finally addressed the issue of linking surface EM measurements with the ground truth from the borehole measurements. While conventional induction logs are only sensitive to $\mathrm{Rh}$, the new generation tensor induction measurements allow both $\mathrm{Rh}$ and $\mathrm{Rv}$ evaluation and calibration because these tools measure both horizontal and vertical conductivity. Thus, they resolve conductive and resistive zones with less bias [31,32].

Figure 2 shows log examples obtained in sand-shale dominated sequences with two new-generation triaxial induction-logging tools from different manufacturers. Both logs show that $\mathrm{Rv}$ exceeds $\mathrm{Rh}$, and the resulting oil saturation is approximately $40 \%$ larger (and hence the reserve estimates are $40 \%$ higher). With these logging tools that yield electrical anisotropy, surface tensor (multi-component) EM measurements can be calibrated and then become more meaningful/valuable and better tied to seismic images. In the absence of modern anisotropy logs, the anisotropy can be estimated from conventional resistivity logs using well-known equivalence principle first suggested by Keller and Frischknecht [33].
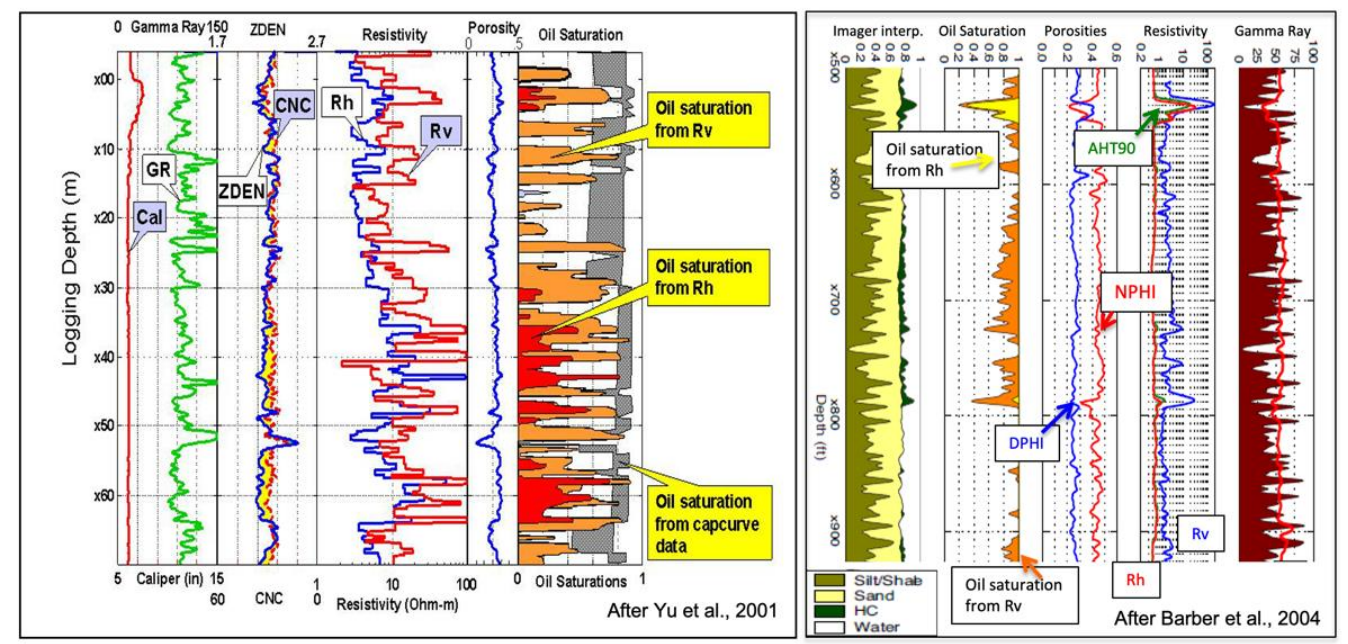

Figure 2. Two data examples of triaxial induction log interpretation using logging tools from two contractors [31, 32]. Here, GR is Gamma Ray log, CNC and ZDEN are neutron and density logs, respectively, AHT90 is a deep induction $\log$, $\mathrm{Rv}$ and $\mathrm{Rh}$ are, vertical and horizontal resistivities derived from the triaxial induction logs, respectively. Both show significant higher oil saturation using the vertical resistivity where they can account for thin laminated shale sequences. Both also show higher Rv than Rh. Here, the oil saturation was calculated from the vertical resistivities, and the increased pay is significantly larger (around $40 \%$ ) than from standard horizontal induction log measurements.

\section{Recent technology advances}

The technical limitation for the use of EM leads to business limitations, and thus, in the past, EM was only applied in selective cases where the value was understood, and the technical limitations were under control [1, 10]. Only after the progress of borehole resistivity logging hardware (Through casing resistivity logging [34] and tri-axial induction logging $[31,32])$, the potential of EM for a sufficiently large application envelope became technically possible $[35,36]$. The value proposition was easier as the reservoir monitoring market is already over 20 billion $\$$, and its value can be expressed in 10-20\% recovery factor improvement. One of the first feasibilities for a real application in the Ghawar field [18] made the limitation in existing hardware clear and strongly pointed in the direction of full integration of borehole and surface measurements.

While a combination of EM and seismic was already proposed by Strack and Vozoff [36], it did not happened until the value of marine EM was proven after 2002 [10, 30]. From 
operational and hardware viewpoint, combining the acquisition of microseismics and controlled source EM lets us use the same technology for reservoir monitoring.

While MT is the standard EM method for basin studies (low resolution due to the low frequency), the higher resolution can be obtained by using a high-power transmitter Controlled Source EM (CSEM) methodology. This is required when looking at fluid saturation changes of oil/ $\mathrm{CO}_{2} /$ geothermal reservoirs and understanding of the reservoir anisotropy is important. Focusing or sharpening of the target image can be obtained by either differential measurements such as Focused Source EM [37, 38, 39] or adding shallow or deep borehole measurements as we describe below.

A broader range of sensors and state-of-the-art electronics yield several fit-for-purpose system configurations and applications that can greatly simplify operations and hardware cost by being tailored to the user's need and experience. For hydrocarbon applications the technology development in this direction is driven by the need to resolve thin horizontal resistors (hydrocarbon reservoirs) requiring electric dipole transmitter and dipole receiver[22-24], [36] similar to the marine EM industry justification [9], [30]. Hence, a grounded dipole transmitter is mandatory. The grounded transmitter produces the transient signal decays more slowly with time and distance than the signal of a loop transmitter and larger signals are a prerequisite for sounding deeper into the earth [36].

To date, the hardware allows measurements better that $0.5 \%$. It still requires extreme care to get the processing of the data to maintain the same error percentage. Once the data is inverted the model assumption smooth the data much more than that and it becomes difficult to maintain that. Hence, it appears unlikely that inversion will be the right tool to be used for reservoir monitoring. It is more likely, that careful imaging where the error propagation is under control will be used.

To validate our development path, we are focusing on commercial projects via feasibilities and field tests. Real checks against logs and comparing data with predictions from 3D modeling prevents following new ideas into dead alleys. It also necessary to calibrate the methods and apply 3D models against real well logs.

\section{Applications leading to technology requirements}

The main areas of application of this technology are in hydrocarbons, geothermal, and in Carbon Capture, Utilization and Storage (CCUS). For hydrocarbon applications, there are two parts of the hydrocarbon reservoir life cycle where EM contributes value: exploration and appraisal/production. The latter focuses more on reservoir monitoring discussed here. Exploration EM measurements are usually integrated with other measurements, and log comparison is done on a large vertical scale (2-5\% of the depth). For appraisal/production applications, the comparison with logs and calibration against the logs is almost mandatory to gage the reliability of the results and to close the calibration loop. The reasons lie in the data usage for immediate operational decisions, rather than long-term drilling decision like in the exploration cycle. For geothermal the application during the exploration stage is like that of hydrocarbons, except that most targets are conductive reservoirs whereas in hydrocarbon applications they are resistive. During the development and production phase the emphasize is on watching the reservoir development and geothermal fluid movement. Once routine operation sets in, monitoring fluids movement and potential induced seismicity is important. For CCUS the knowledge of state of the reservoir and the tracking of the injected $\mathrm{CO}_{2}$ is important. The state of the reservoir mostly refers to seal integrity and observing seal breakage [16]. Fluid movement can be tracked once sufficient fluid has been injected. Careful feasibility 3D modeling is strongly recommended supported by on-site noise measurements with the system that is used for the survey. Below we will give an example for that (for a geothermal application).

During the exploration cycle following are the main applications of EM:

- $\quad$ Sub-basalt and sub-salt imaging where seismic have difficulties to penetrate

- Imaging below over-thrust (difficult for seismic) 
- Mapping of lateral fluid variation in carbonates

- Mapping high resistive oil reservoirs in a sedimentary section

- $\quad$ Mapping coal seam sequences

- $\quad$ General depth to basin mapping

- Complex and deeply weathered near surface penetration

- Mapping of geothermal higher temperature zones (more conductive zones)

- Geothermal anomaly and low-density zones mapping in combination with gravity

For the appraisal/production cycle, the applications are:

- $\quad$ Mapping of water/steam fronts

- Unconventional reservoir depletion mapping during initial production

- Hydro-fracturing mapping

- Defining sweet spots in reservoir by mapping of lateral fluid variations

- Upscaling of borehole resistivities to reservoir scale and lateral dimension

- Defining sweet spots in the water aquifers for secondary water production

- Mapping field extend and supporting in-fill drilling

- $\quad$ Supporting geo-steering applications

Figure 3 shows the survey layout for exploration (top) and monitoring (EOR, geothermal, or CCUS) (bottom) applications. 2D lines which include receivers recording all EM components would be usually used for basin studies, while 3D layouts (top figure on the top right) are more directed to define a detailed drilling plan or image below basalt, sub-salt and overthrust. The monitoring layout at the bottom of the figure shows an example for water-flood monitoring but the model for geothermal or CCUS would be similar. Complex terrain applications are common in the US unconventional plays where the equipment gets deployed by helicopter (as in the mountainous part in the figure). All of this leads to immediately the requirement of low power (long operating time), system stability against drift and external magnetic field noise and a multi-channel concept to be able to deploy many units. Making the system scalable also leads to start/stopping of the system by a push of a button and starting with a pre-defined acquisition sequence. For reservoir monitoring we often want to add more sensors, and the use of sub-acquisition controller that record the data in the main node appears to be cost efficient. From operational viewpoint, turn-around time and fast equipment movement is important. It can be addressed by adding Cloud data delivery and artificial intelligence to the operations [43].

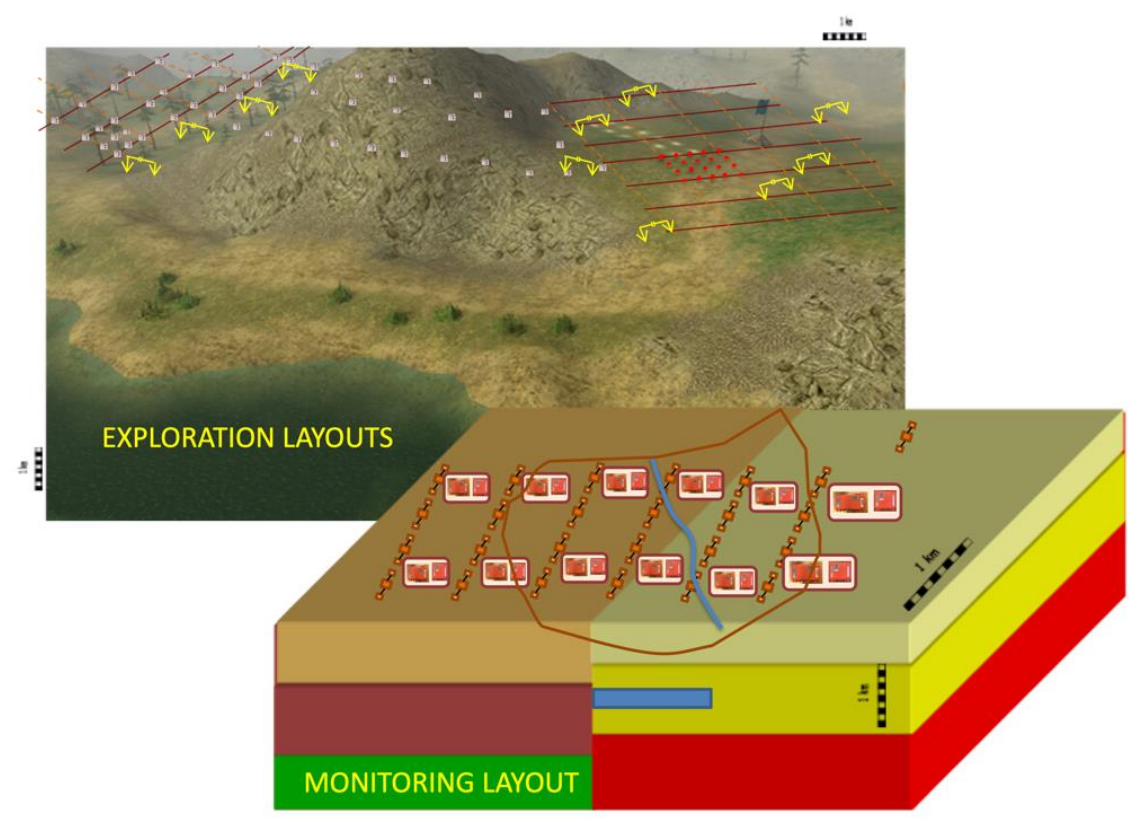

Figure 3. Survey layout for using land EM system for exploration and monitoring. The top shows 3 types of layouts: on the right a 3D layout where data is acquired with an aerial layout and sorted 
into bins with only ONE full magnetic field component site per bin (since the magnetic field does not vary much laterally). In the middle are sites laid out over complex terrain sometimes deployed via helicopter. On the very left of the top diagram are several 2D lines where each site has all EM components. At the bottom of the figure is a model showing a reservoir that can simulate a scenario where the fluid movement across the reservoir boundaries is to be monitored via repeat measurements. The model is typically a 3D anisotropic model as described in the text.

Since field operations are the biggest cost factor driving the hardware implementation, we will review standard configurations for MT, CSEM, and reservoir monitoring. We classify into these categories because standard MT and CSEM are mostly focused on detecting subsurface resistivity structure while reservoir monitoring is focused on timelapse or repeat measurements. The former is a more interpretative process that requires integration with other data sets and often with inversion while the latter requires more detailed attention to very accurate measurements and to changes of the system to translate the accurate measurement to 3D changes in the resistivity structure (and thus fluid content) which need to be mapped. While the equipment for all the methods is the same, the processing workflows are very different. Also, for time-lapse measurements highly accurate and repeatable measurement are required with the minimum processing needed.

We have tried to simplify these workflows and hardware and to make the entire technology look closer to a seismic system (user friendly). Figure 3 shows the overall operational survey plan with the site layout shown in Figure 4. We designed our array system with these requirements in mind [44]. Shown are a broadband MT site on the top left with various other MT configurations that are used for various purposed to obtain better images.

\section{Magnetotellurics (MT)}

MT measurement typically record 5 components (3 magnetic and 2 electric field). Detailed description of the MT method is given by Vozoff [3, 36, 37], Simpson and Bahr [48], and more recently by Chave and Jones [49] with more practical aspects of the implementation for the geothermal industry [50,51]. A MT site layout is shown at the top left of Figure 4. The frequency bands of MT can go from approximately $10^{-4}$ to $10^{4} \mathrm{~Hz}$ and is commonly divided into:

Long period Magnetotellurics (LMT) $10^{-4} \mathrm{~Hz}$ to $0.1 \mathrm{~Hz}$

Broadband Magnetotellurics (BMT) $-0.001 \mathrm{~Hz}$ to $1000 \mathrm{~Hz}$

Audio Magnetotellurics (AMT) $-1 \mathrm{~Hz}$ to $20 \mathrm{kHz}$

Figure 4 (top left) shows the system building blocks and includes a very low frequency ( 0 to $180 \mathrm{~Hz}$ ) fluxgate magnetometer which we term as extended LMT sensor. Modern systems include broadband coils from $2.5^{*} 10^{-4} \mathrm{~Hz}$ to $10^{4} \mathrm{~Hz}$ bandwidth, which is a cost saving (operations and asset) over the traditional BMT systems. To achieve maximum range for MT systems, fluxgate sensors are added which are the premier choice for deep crustal studies. Fluxgate sensors are also less noisy below $0.1 \mathrm{~Hz}$ compared with induction coils because they measure magnetic field variations directly compared to field derivative measured by induction coils (like borehole induction tools). Induction coil sensors above $0.1 \mathrm{~Hz}$ are less noisy than fluxgate. Obviously, engineering implementation can modify that to a certain degree but cannot completely overcome physical limitations. It is used to match sensor to desired frequency range. An example of various sensor 
spectra is given in Figure 5.

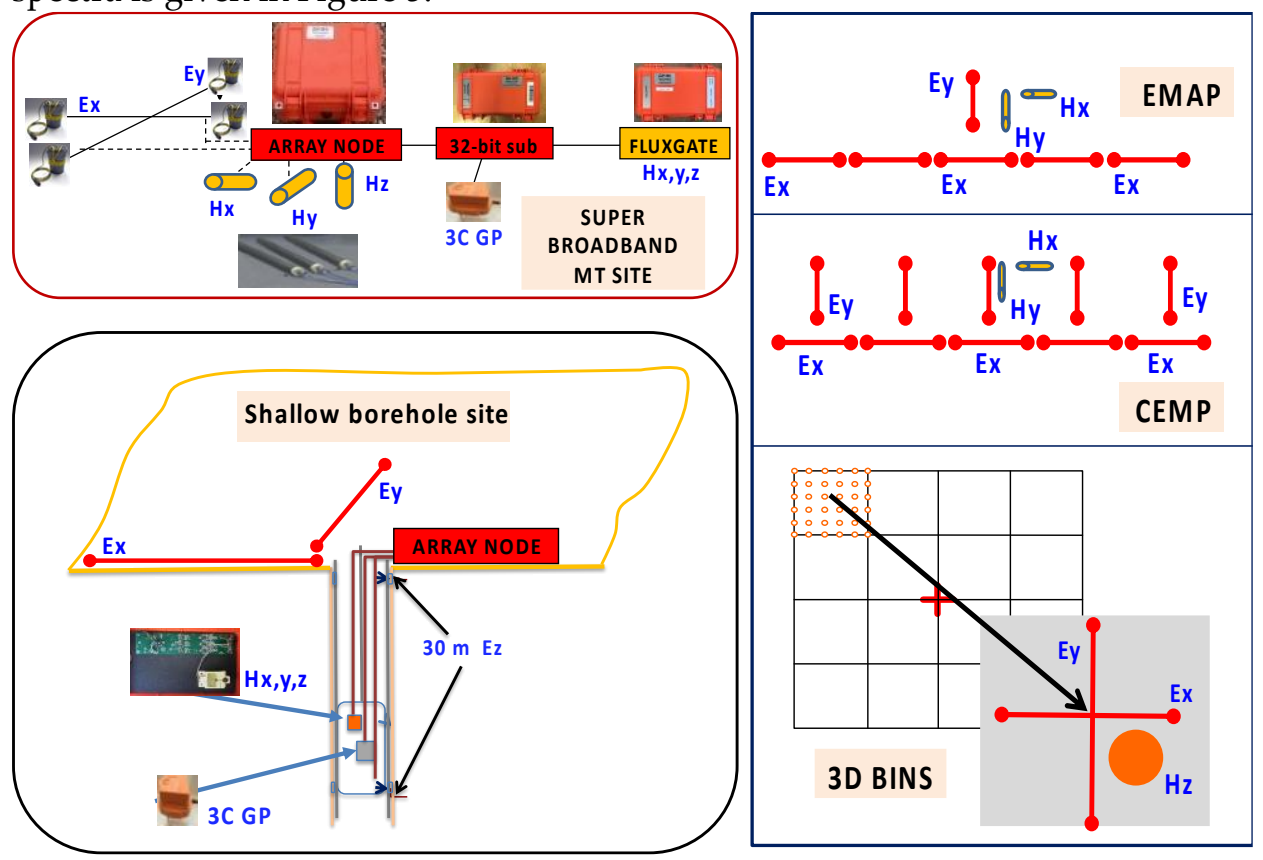

Figure 4. Site layouts block diagrams for magnetotellurics (MT) on the left top, shallow borehole seismic/EM on the left bottom, electromagnetic array profiling (EMAP) [35] on the top right, continuous EM profiling (CEMP) right below. The bottom right shows a zoom of the 3D array layout when the data gets acquired in bins.

Together with standard MT, the electrical mapping was fashionable during 1980s and 1990s to understand the effect or near surface statics [45]. It enabled to better tie the data to seismic results (EMAP - Electromagnetic array profiling). Later, after 2000, perpendicular electric fields were added, and the term CEMP (Continuous EM profiling) was coined [52]. This gives additional structural control and results in more stable 2D inversions. Around 2010, 3D bin acquisition was used. It is based on the magnetic field, which varies little laterally while the electric field varies a lot. Thus, only sparse magnetic field can be used (saving hardware and operating cost), specifically one full magnetic field set per bin. These magnetic fields are used for all the electric field sites in the same bin. As input to the system requirement this means that for many electric field data points you would want remote sub-acquisition controller so that the channel count for each main node gets increase. This can be achieved by adding these digitally. MT depends on very accurate phase measurements, so including a stable digital connection between the main node and a 32-bit sub-acquisition controller require careful phase considerations (see Figure 4.). Wireless transmission is possible, but any data transmission should be kept away from analoge parts of the system. This helps to avoid potential noise from the wireless transmission and saves a significant amount of operational cost [43].

The sensors are the most important part of the system for all methods. Choosing the right sensors means understanding their limitations. The electric field sensors need to be long-term stable (2 months) with a grounding resistance around $1 \mathrm{kohm}$. New EU regulations require to use lead-free electrodes; therefore, majority of manufacturers provide appropriate sensors with marginal difference in quality. For MT and monitoring, the longterm stability is essential, while for frequency or time domain CSEM less expensive electrodes can be used. In arid regions, regular watering with a copper-sulfate (or any other saline) solution may be required. For magnetic field measurements, the sensor selection is related to the applications and cost (operationally also to the size). While we always try using the same sensor for the vertical magnetic field measurement, it can be replaced by a shorter one or by air coil when terrain or hardness of the ground requires it. While bandwidth and depth of investigation couple via the skin depth, you do get better fidelity data 
by acquiring at least one decade higher and lower frequencies than what is required by the target.

Figure 5. shows the comparison of typical noise densities for magnetic field sensors for land and marine applications. We show noise densities of standard MT coils (Lemi118, Lemi-120, Lemi-152), one optimized for earthquake prediction coil (Lemi-030) and a shorter MT coil for marine (or land) applications (Lemi-121). They are compared with fluxgate sensors (Lemi-025, and LEMI-029). Note the cross-over in the noise densities between fluxgate and coils around $0.1 \mathrm{~Hz}$ or 10 seconds. Below that frequency the fluxgate sensor is usually less noisy than coils. One can extend the range of fidelity for both coils and fluxgate sensors and in fact that is being done in the recent broadband sensors by several manufacturers.

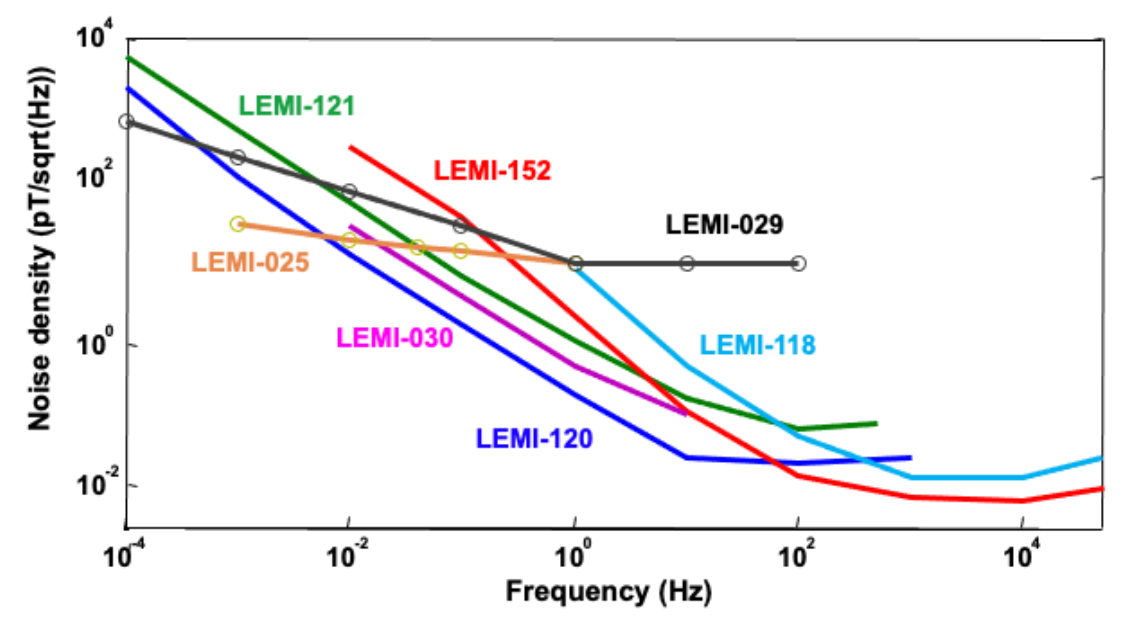

Figure 5. Examples of noise density spectra for typical land and marine sensors comparing standard induction coils and fluxgate magnetometers.

Figure 6 shows a comparison between fluxgate sensors and inductions coil in normal measurement mode and when we extend the usable fluxgate range. The range extension is achieved when we use the electric field (normally the magnetic field is used) to calculate the admittance tensor. This works well in most cases where the electric field is less noisy than the magnetic field. The top of Figure 6 shows the apparent resistivities in both orthogonal directions for the data measured at the same location with fluxgate and induction coil sensors. Note that from $0.1 \mathrm{~Hz}$ down (period $10 \mathrm{sec}$ up) the fluxgate response gets stronger (similar as in above figure). Using the electric field for the admittance we can extend this range in the upward direction to almost $10 \mathrm{~Hz}$ (period $0.1 \mathrm{sec}$ ). This is important because fluxgate sensor cost is approximately one third of that of induction coils. 


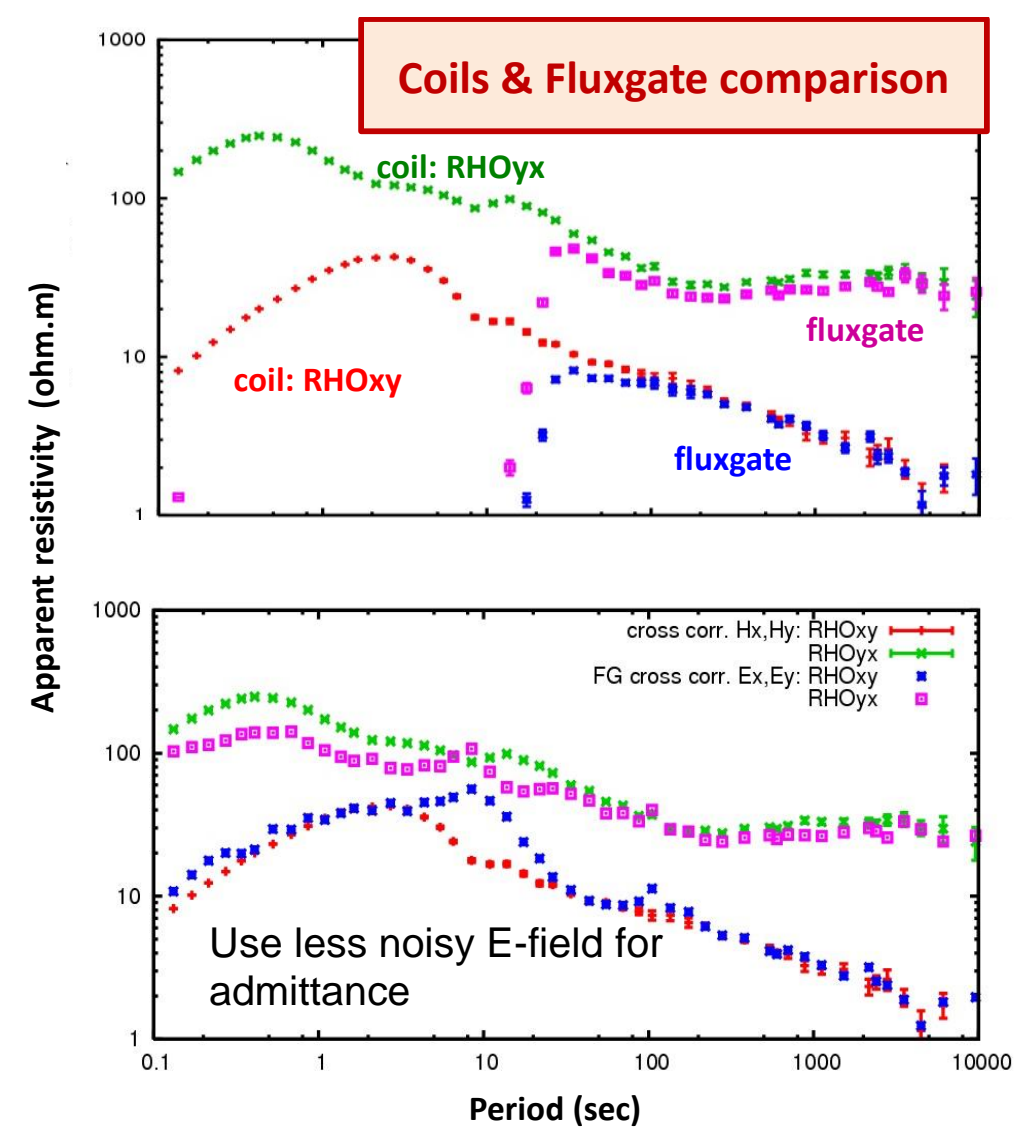

Figure 6. Examples of optimized fluxgate sensors processing. The top graph shows standard processing for induction coil and fluxgate data. The top graph clearly shows that the fluxgate data become noisier for short periods below 10 seconds $(=0.1 \mathrm{~Hz})$. At the bottom, we apply a different admittance calculation using the electric fields and can in most cases extend the reliability.

\section{Controlled Source Electromagnetics (CSEM)}

In the past there were two periods of increased activity in CSEM, one in the 1950s and one in the 1980s [1]. The technology of the 1980s is still being used to a large extend, while substituted with modern more stable electronics, and GPS has been added. While MT became the workhorse of the geothermal industry, CSEM methods were only used on experimental basis. One of the reasons lies in the difficulties of reconciling the results with well logs which in the past mostly gave horizontal resistivities. Since sedimentary environments are anisotropic, as mentioned above, this is a serious limitation. CSEM used predominantly a grounded dipole transmitter and is sensitive to both vertical and horizontal resistivities. Until the event of tri-axial induction logs [53], which allow measurement of the vertical resistivity, correlation could only be inferred using equivalence methods described by Keller and Frischknecht [54, 55]. Today, we can correlate/link surface measurements with vertical resistivities and borehole measurements (both with horizontal and vertical resistivities) using the suggestion by Keller and Frischknecht [54]. This is a valuable tool in correlation and linking vertical and horizontal resistivities from various data sets and defining all possible solution scenarios.

Another limiting factor is the information focus. Since we use a transmitter and a receiver, the information, in principle, comes from the volume between the transmitter and the receiver. Putting the information at the right place is always an interpretative skill, hindering the widespread use of the technology. We will discuss below that we can overcome this limitation by focusing the information, that will allow us a new evaluation of CSEM onshore methodology with modern hardware and software implementation.

CSEM is divided into frequency domain and time domain methods [56]. Frequency domain methods utilize a sequence of individual frequencies, and the same frequency 
data are then observed, and amplitude and phase between the received and transmitted signal are measured. Time domain methods utilize turn OFF (or ON) of a current, and we observe a decaying signal with broadband frequency content. To explore the depth of 2-3 $\mathrm{km}$, time domain methods have prevailed within the last decades [1], [55 - 57], especially on land / onshore, mostly because you record the signal in the absence of primary field and we can thus observe small voltages easier. With the merging of new hardware technologies time-domain and frequency-domain measurements are very similar from measurement viewpoint. The remaining difference becomes the energy focus, namely in time domain entire transmitter energy is focused on a limited volume for each time step while in frequency domain the energy distributes on the entire volume between transmitter and receiver.

Controlled source EM frequency domain methods follow the same hardware consideration as MT for survey setup and receiver layout. Distances between transmitter and receiver are usually larger that 3-4 times the exploration depth. The advantage over MT is that now each component independently can provide the subsurface resistivity structure, and joint inversion of mixed components provide inside into the geology. Frequency domain (onshore) methods are common for shallow depth $(<500 \mathrm{~m})$ except when integrated in Time-Frequency-EM (TFEM) as described below. For marine applications frequency domain CSEM is almost exclusively used [30].

Time domain methods as described by Keller et al. [57] and Strack [55], use a grounded dipole transmitter and multi-component receivers to acquire the electric and magnetic fields. Offsets are usually comparable to target depth, though shorter and longer offset can be used. This method is also known as Long offset transient EM (Lotem) [58] referring more to the grounded dipole transmitter than 'long', which was a historic requirement when amplifiers did not have sufficient dynamic range and so longer offset were required to squeeze the dynamic range. This requirement completely disappeared with the introduction of floating-point seismic technology standard into the acquisition units [59]. Today, with 24/32-bit acquisition technology this is no longer a consideration.

To reach a depth from $500 \mathrm{~m}$ to at least $3 \mathrm{~km}$, the transmitter power is usually 100 kVA or above ([57] used $1 \mathrm{MW}$ ) and thus costly to operate. Thus, it makes sensors to layout at as many receiver locations as possible. Cost considerations on the sensor side become quickly important. In time domain CSEM, each component, like frequency domain EM, gives complete information. One can often live with just the vertical magnetic field, which can be measured using a multi-turn air loop. This air loop can be constructed out of multi-wire cable, and it is many times less expensive than induction coils. Obviously, if surface area is limited, coils or fluxgate magnetometers can be used. It is important that the sensors are as broadband as possible. The compromise between acquiring a full multicomponent, multi-directional data set and optimizing cost can easily impact the target resolvability. Thus, we always advise to carry out feasibility studies and even noise test (in noisy areas) to optimize the layout. From our experience of 40 years, surveys with careful pre-survey feasibility studies were mostly successful.

In the past, large transmitters were used [57] on special occasions. With the improvement of receiver electronics and noise reduction methods, the transmitter power could be reduced, as the received signal is directly proportional to the transmitter current. We are today at a stage where for the deep hydrocarbon $(2-3 \mathrm{~km})$ and geothermal applications a transmitter of 100 to $250 \mathrm{kVA}$ is sufficient versus $500 \mathrm{kVA}$ to $1 \mathrm{MVA}$ during the 1980s. Long term stability of the transmitter in terms of the current and the waveform is paramount for time-lapse measurements, and for reduction of data processing time. For this reason, we choose the lowest voltage and lowest inductivity of the transmitting dipole. Safety (fires and electric shocks) is highest concern, and careful grounding and ground separation must be considered as the transmitter dipole injects current in the ground which can flow back to the generator. Figure 7 shows a pictorial layout of the transmitter components with a photo of a transmitter site. A typical controller setup is on the top left of the figure. Here, the transmitter parameter is recorded, the waveform is generated, and transmitter dipole safety is monitored. Safe, controlled, continuous operation is of 
paramount importance as any shutdown will cause significant additional efforts in data handling and processing. It is advisable to use as many pits as possible for grounding transmitter electrodes and to keep them well watered because it reduces the current flow through each pit and prevents disintegration of the electrodes. The transmitter must be GPS controlled and timing differences between GPS timing, acquisition system timing and current waveform synchronization must be accounted for. Current injected via dynamic electrode pits always varies and for monitoring applications constant current control to better than $0.5 \%$ is mandatory. In more resistive environments and shorter offsets, the system response should also be monitored for each current switch as well as shorter switching times may be necessary. The heart of the layout is the switchbox in the center.

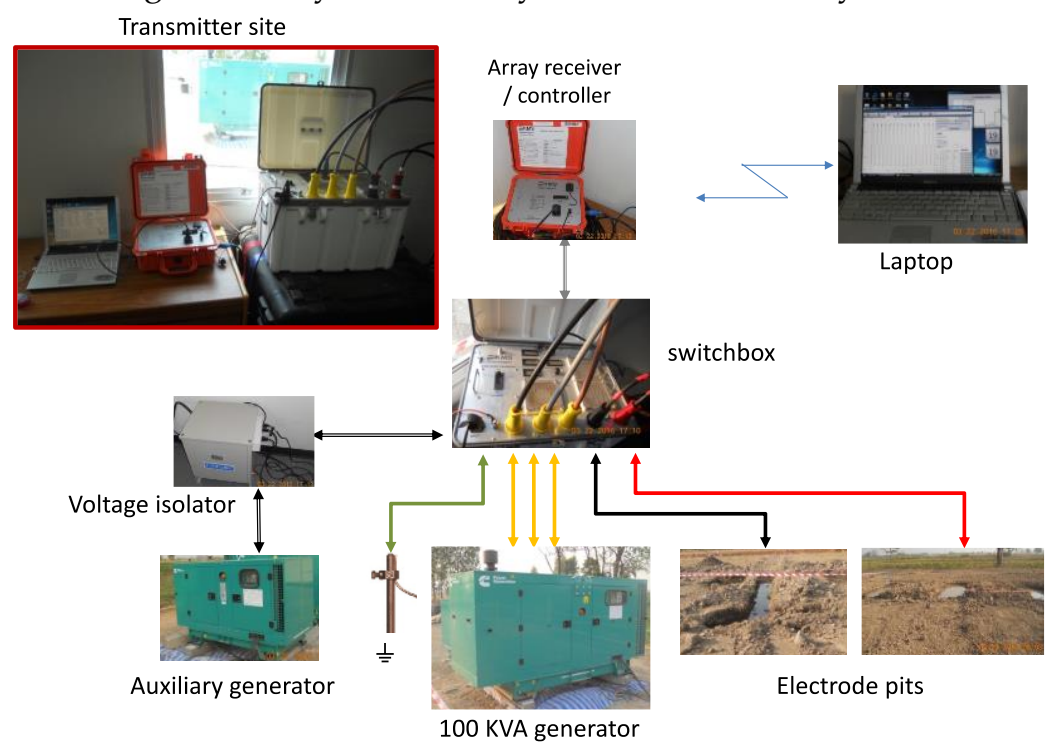

Figure 7. Pictorial transmitter component layout. A transmitter consists of a power source (generator), electrode pits connected to the generator by cable, a switchbox that rectifies the current, switches it and controls electronics. One set of the switchbox is powered by an auxiliary generator (to maintain control when power generator fails). The system is controlled by the array receiver, and data are monitored in real time.

Here, the current is rectified and switched according to the waveform provided by the controller (array receiver/controller in the figure). The switchbox also includes multiple safety circuits for inputs and outputs and allows the controller to monitor current and initiate additional safety protocols. To ensure that no ground loops occur the power injected into the transmitter dipole and its control electronics are electrically isolated and the control electronics is driven be a separate power source (auxiliary generator in the figure to be able to turn on and off) and connected via an isolation transformer (to avoid incidental grounding via laptop chargers etc.). A clean grounding concepts is important to avoid incidental shock at the transmitter site. For details on the electrode pit preparation, we refer to [55].

Historically, transmitter switching times depended on the inductance of the grounded dipole (approximately $2.5 \mathrm{mH}$ per $1000 \mathrm{~m}$ wire) and the switching electronics. Today's high-power switches switch instantly and can turn a current on or off better than 1 micro-second. The more abrupt the current gets switched, the more ringing we see due to the Gibb's Phenomenon. This ringing can cause problems for our data processing in timing synchronization. For reservoir monitoring repeatability and stability is key to see small reservoir parameter changes with time. Since measurement accuracy better then 0.5 $\%$ is easily achievable, we must push system accuracy and repeatability to below that as processing hardly gets below the 3-5\% error threshold. We thus choose a depth target range from $500 \mathrm{~m}$ to $6,000 \mathrm{~m}$ and a resistivity range from 2 to $10 \mathrm{ohm}-\mathrm{m}$ (as rule of thumb $95 \%$ of hydrocarbon reservoirs rock resistivities (terrestrial) are between $3-30 \mathrm{ohm}$ ). This will give us a wide range of signal time windows which are mostly longer than $700 \mathrm{msec}$. 
We thus add control electronics to keep the ramp time for switching just below 1-3 ms to minimize current ripple due to switching. Using different switchboxes (100 and $150 \mathrm{KVA}$ ) and transmitter locations in USA, China, and Thailand where the dipole cable had different length and inductances, we observe stable switching times more than 10 times shorter than the uncertainties produced by processing. If we address shallower target and work in much higher resistivities like in crystalline rock, we can field-adjust the ramp time.

Since many practical and safety issues are addressed in [55], we will select here an issue affecting the repeatability of the measurements which is a key issue to use EM for reservoir monitoring. We mentioned above that today's electronics can make many times more accurate measurements than we require. System manufacturers try to limit themselves to $0.5 \%$ (for business reasons), yet state-of-the-art systems can do more than 10 times better. This means that we must get those parameters that are inferred better than that accuracy level. The transmitter current is such a critical parameter. Apart from the switching time discussed above, we must also operationally control it by monitoring it and its waveform. Figure 8 shows an example of the ramp times at different phases of the processing. On the top left of the figure is the original current recording including the typical AC ripple during current injection. Below on the left is a zoom of the ramp time showing a consisting $2 \mathrm{msec}$ ramp with very little ripple. On the right of the figure, we compare two different waveforms (50 and 100\% duty cycle) for a different transmitter. Both duty cycles are stacked data and below is the system response recording done $1 \mathrm{~m}$ next to the transmitter cable using electric field sensors as used for the survey. This system response includes the cable-to-Earth coupling and receiver electrode coupling. For the bipolar waveform it is $70 \mathrm{msec}$ and for the reversing polarity it is only $30 \mathrm{msec}$. This can be explained in such a way: By reversing polarity you can overcome the ringing by switching in the opposite direction and while the ringing still exists, it become negligible when the current amplitude increases in the other direction. Here, it becomes important to note that the actual switch can be faster than 1 micro-second and we are slowing them down to control the ringing to maintain a consistent current waveform. We also notice in the stacked currents on the right that there is a small drooping of the current despite current control. This is cause by the electrode pit's electrochemical reactions. For data processing this means that we must normalize the time series by the current for each sample. All of this in addition to long recording times is necessary to obtain reliable, repeatable measurements with an accuracy better than $0.5 \%$.
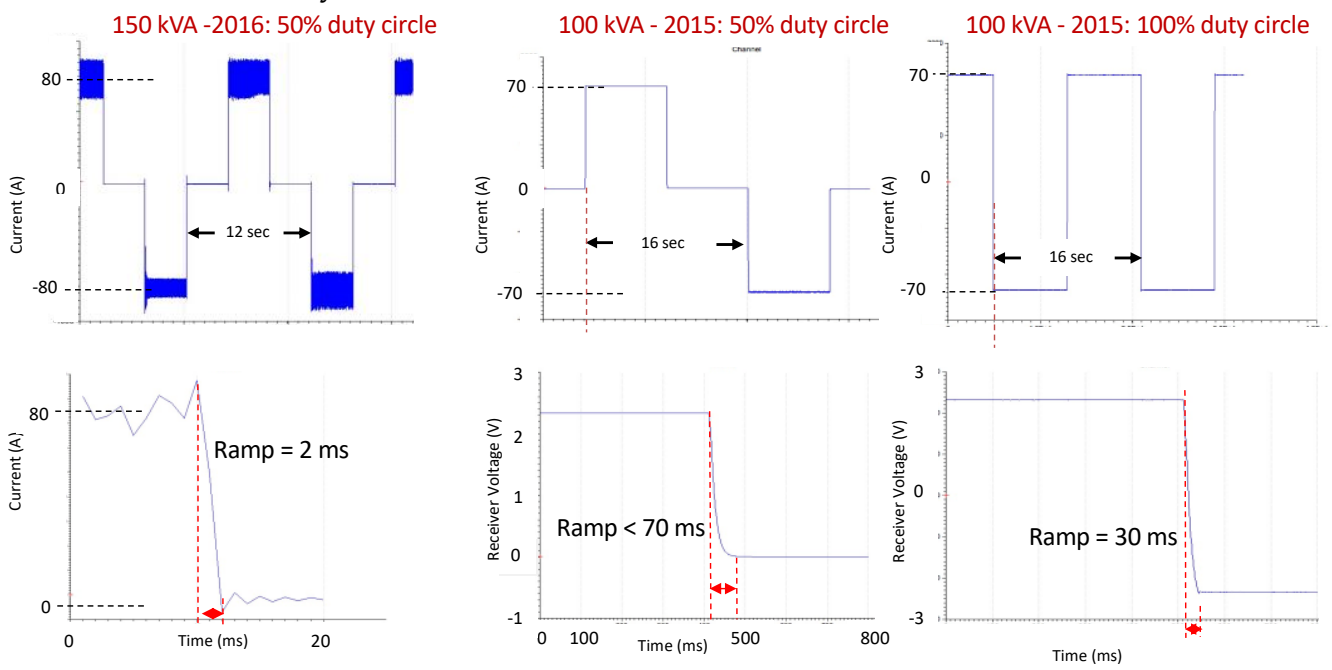

Figure 8. Examples of current recordings for different data stages. On the left we have an original current recording with a zoom display of the turn-off below. In the center and on the right, we have stacked current time series for bipolar (50\% duty cycle) and reversing (100\% duty cycle) waveforms. Below the current waveforms are the signal recording by electric field receivers placed with 1-2 m next to the transmitter wire. 
As mentioned above, one of the challenges to EM methods is the information focus. We address this by using differential measurements known as Focused Source EM (FSEM) [37-39, 60, 61] and adding shallow/deep boreholes to the system [36]. FSEM methodology is described in the following Figures 9 and 10 and an example to map fluid in carbonates in given in [39]. The FSEM configuration works like focused borehole laterologs [62] and uses differential measurements. The differences between adjacent receivers are subtracted and appropriately normalized to only produce a sensitivity to the vertical electric field between the receivers.

Figure 9 shows on the left the sensitivity (2D) for different receiver offsets for frequency and time domain. In the frequency domain, we sample the entire volume between transmitter and receiver, while in the time domain we are sensitive to a volume below the receiver and a volume below the transmitter. If we apply either frequency or time domain FSEM technique, we obtain mostly sensitivity below the receiver as depicted on the right of the Figure 9 where we are left with the volume between the receivers after subtraction.

Following, we consider first a reservoir example and then look at a field test. For a realistic example, we calculated a 3D model for the different CSEM methodologies using a 3D EM modeling software CSEMulator [63, 64]. Figure 10. shows the response of two $3 \mathrm{D}$ reservoir targets at $2.5 \mathrm{~km}$ depth. It can simulate a hydrocarbon bearing reservoir and a $\mathrm{CO}_{2}$ storage reservoir as they are resistive. Both time- and frequency-domain Controlled Source EM methods give an anomaly of 10 and $40 \%$ for the small and large reservoirs, respectively. Using the FSEM method shown on the right, the same reservoirs produce a significantly larger anomaly of 200 and 40\%, respectively, with clear separation between them. Another benefit of applying FSEM is that it removes near surface effects between the transmitter and nearest receiver because it considers mostly the differences between the receivers. We found this to be true in most, but not in all cases that we considered. The original FSEM methodology was derived for multiple transmitter positions, but for real field operations this is sometimes difficult from logistics/cost viewpoint. We thus modified the methodology and with simple cable adaptors implemented the method for our array

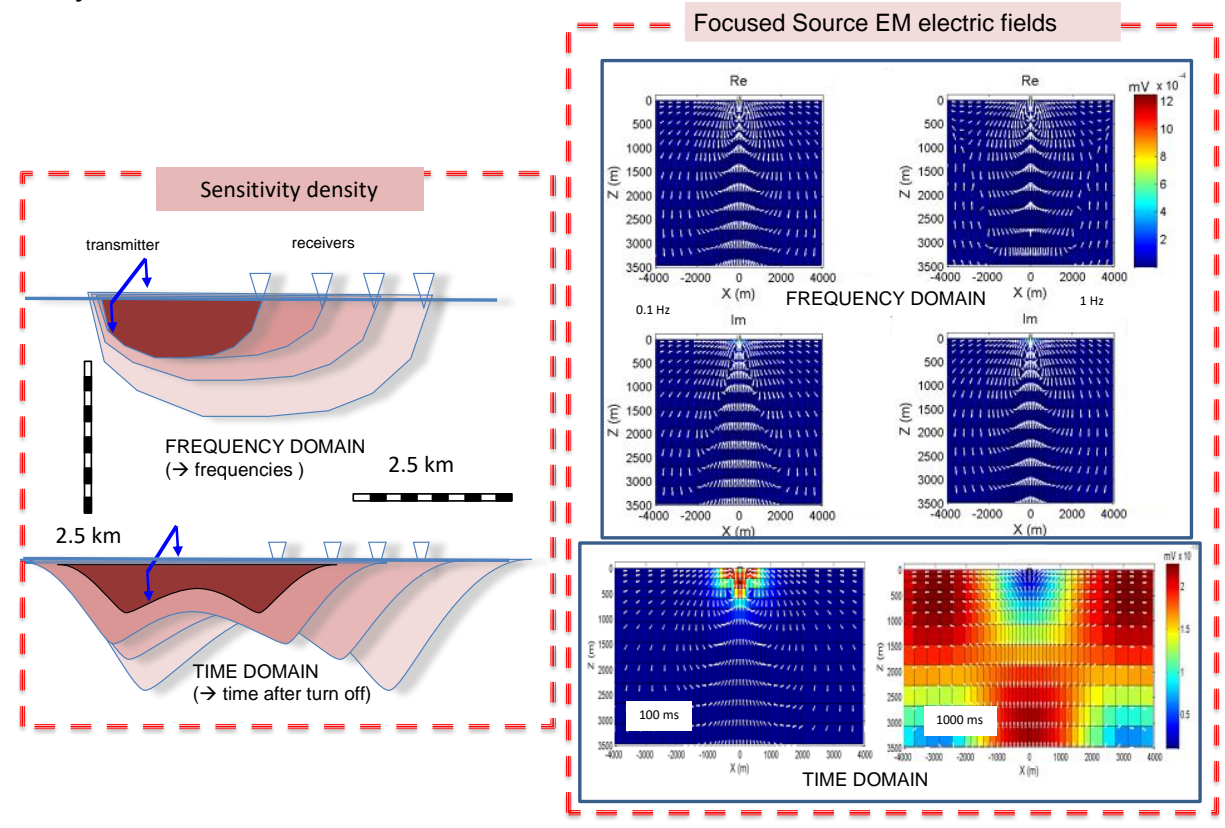

Figure 9. Summary sensitivity plots for time and frequency domain on the left and for Focused Source EM on the right. For the frequency domain (top left) it shows that with increasing offset we have different volumes of investigation for different frequencies. Similar in the time domain (bottom left) except that the sensitivity is focused around two maxima (one near the transmitter and one near the receiver). For both domains, when we take the difference between two receivers, we subtract the effects of the horizontal currents (between the transmitter and nearest receiver). We are left with the 
difference information content coming from the difference volume. This is equivalent to the current flow underneath the receiver (after [39]).

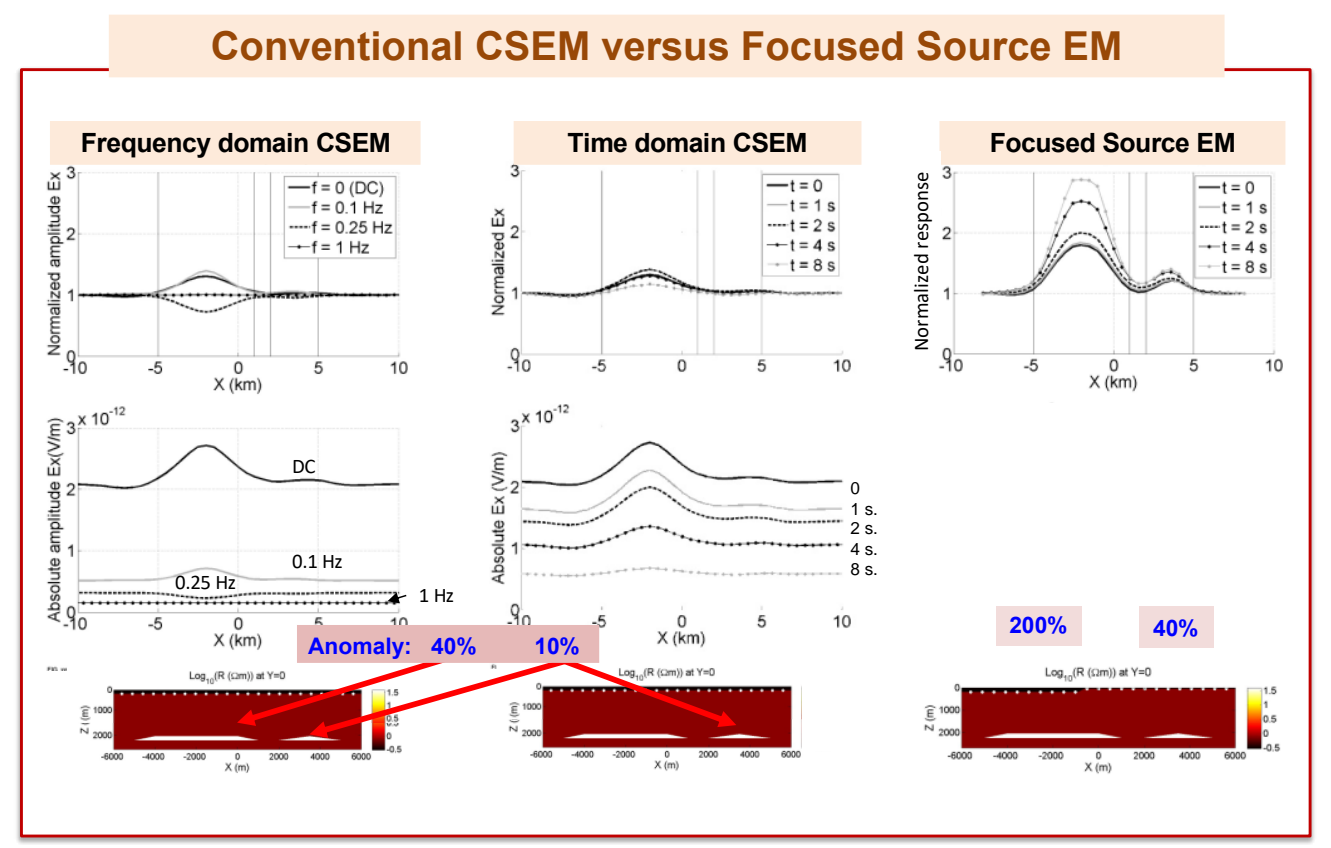

Figure 10. 3D modeling results for $3 \mathrm{~km}$ deep reservoirs (larger and small) in frequency (left) and time (middle) domain, and Focused Source EM (right). Note that for both frequency and time domain the anomaly is approximately $40 \%$ for the large target and $10 \%$ for the small one. For FSEM the anomaly is $200 \%$ for the large reservoir and $40 \%$ for the small one.

The test site was near Houston at the edge of Hockley salt dome. The data were processed using the software workflow described below [65], inverted with 1D (where possible), imaged with various algorithms, and subsequently modeled in 3D. Figure 11 shows a compilation of the 3D model that matches the data. The two cross-sections are extracted from the 3D model. The data plots below show the match between modeled response (dots) and field data (solid lines). On the left there are the electric field transients from three electric field receivers $\mathrm{Rx} 1, \mathrm{Rx} 2, \mathrm{Rx} 3$, in 2 directions. On the right there are the FSEM data curves versus the modeled responses. Both fit very well. The model was constructed using the anisotropic 1D (horizontally layered) background model derived from an available resistivity well log. The modeled resistive body on this background (representing the salt dome with resistive caprock above it) was composed of seven parts or segments: under Tx-North, under Tx-South, under receivers $\mathrm{Rx1}, \mathrm{Rx2}$, Rx3 and two flanks. Upper part of each segment was modeled as a trapezoidal prism as depicted in Figure 10 (top). Geometry parameters of each prism and of underlying shapes, with optional overhangs, varied using model-compare-update (MCU) approach as a simplest parametric 3D inversion. It was done manually since this case is too special to develop automatic routine. MCU approach was the fastest way to get the answer due to (1) a moderate number of the unknown parameters; (2) visual interpretation of FSEM data: since the circular dipole is sensitive to vertical currents, we can directly see where we have stronger/weaker vertical current flow; (3) high speed and efficiency of the forward modeling. While more data are needed to be acquired to fits the entire salt dome, the match is already remarkable. Haroon [66] applied another implementation of FSEM for shallow saltwater intrusion mapping offshore Israel and reported an improvement of the imaging results. 

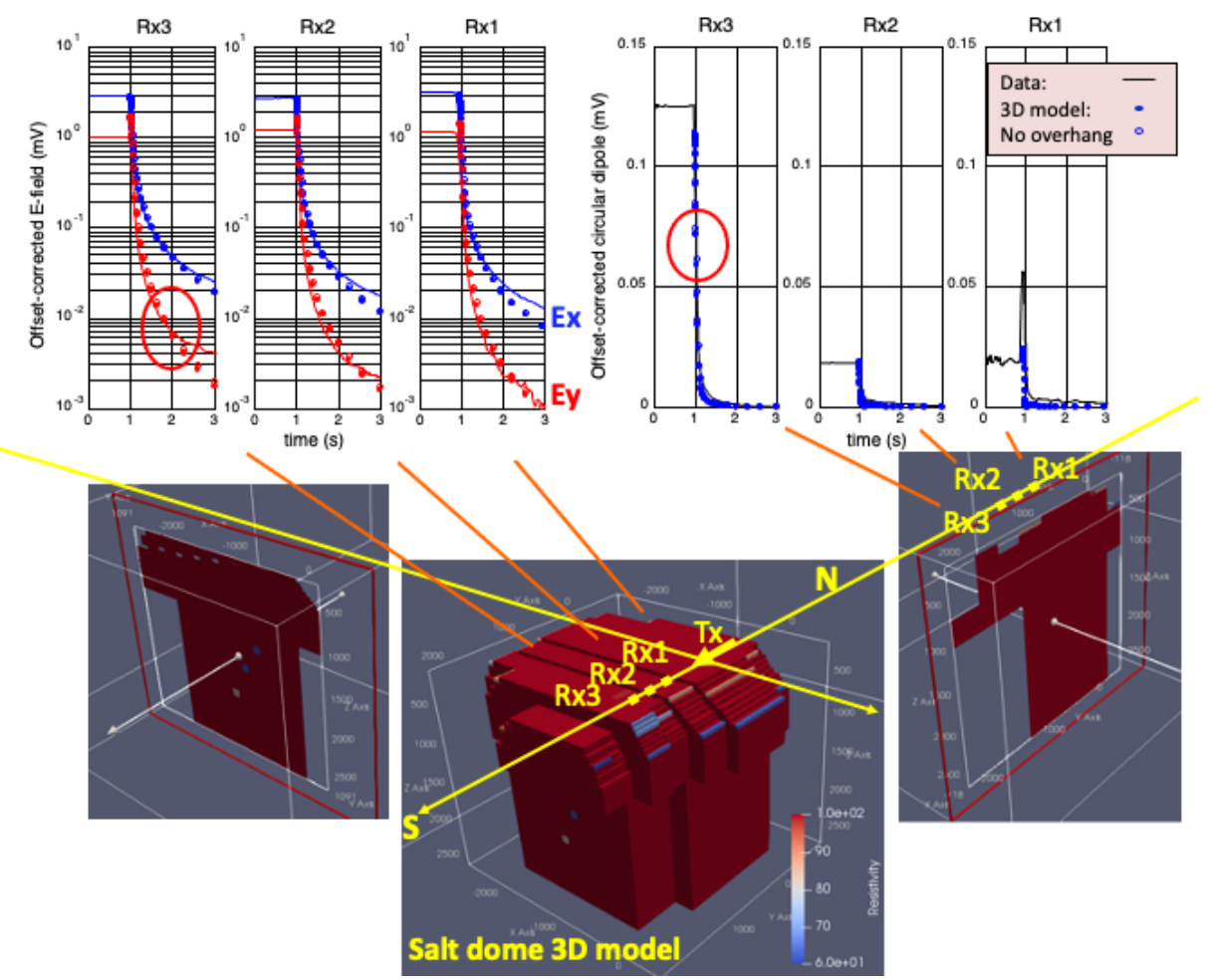

Figure 11. D model resulting from interpreting FSEM measurements over Hockley salt dome near Houston, Texas. Also, two section slices are shown as indicated in the model. At the bottom of the figure, we have the 3D model response displayed. The electric field transients are plotted versus the 3D model responses on the left, and the FSEM data versus model on the right.

Time-Frequency-EM is mostly used by our Chinese colleagues as a routine method for mostly hydrocarbon area when the detailed resistivity structure is not known. Thus, it essentially combines all CSEM methods into one including induced polarization [22, 23, 52]. The acquisition workflow is automated and all or a sub-set of the EM field components is acquired. With a grounded dipole transmitter, first, time domain responses at various frequencies are recorded one after the other. Then the individual frequencies are scanned through sequentially, and finally the induced polarization response is recorded. Processing can be done in time domain or frequency domain. Inversions are done individually or jointly. In a joint inversion the time domain response would provide the volume focus and the thin resistor (reservoir) anomaly, while the frequency domain would give full volume sensitivity and the induced polarization value and static effect calibration. Finally, the induced polarization would show any anomalous charge accumulations often associated with hydrocarbon reservoirs. In term of the data production there is an advantage as the careful survey design is avoided. Also, we are avoiding the bias towards conductors or resistors inherent to each field component. The cost is more data and more processing. The drawback lies in the lack of fine tuning of the survey parameters to improve target resolution. Obviously, 3D interpretation is difficult for this large data combination and will smooth the target response. The array system, discussed here, has a scheduler which can work through all these sequences automatically and change the acquisition setup accordingly. This includes changes in the transmitter setup.

We discussed survey setups to improve the imaging and addressed the difficulty with the image focus. Why can we not measure directly as voltage what we have modeled for FSEM? Unlike in the borehole where voltages are in the nano-Volt range, we can do this for surface measurements by observing the vertical electric field. It gives us directly the current flowing in vertical direction to the receiver as the 3D modeling results show in Figure 9 on the top right. It is sufficient to go just below the surface and we built a sensor package - a small/shallow borehole receiver- that allows seismic shot holes to be used for its placement ( 20 to $30 \mathrm{~m}$, extendable). It uses also surface tensor electric field 
measurements to compensate for any potential misalignment of the vertical electric field. The components diagram and field setup are shown on the left bottom of Figure 4. Figure 12 shows a typical 3D anisotropic model for a monitoring setup with several shallow borehole receivers. Picture of the transmitter site and shallow borehole tools are shown in the figure (top left) and the shallow borehole receiver on the top right. We display the anisotropy ratios as they common in many sedimentary basins. Objective is to monitor the flood front. Figure 13. shows the modeling responses for inline electric field and the vertical electric field measured in the shallow borehole. At the bottom center of the figure the FSEM response is shown. The curves are displayed for different times after turn-off along the profile line. The anomaly due to the water flood for the inline surface measurements is $1.5 \%$, for the vertical electric field in the borehole $>10 \%$ and for FSEM at the surface $8 \%$. Note there are some shifts of cross-over and maxima with respect to the distance from the water flood front. This means that 3D modeling/inversion will be required for more accurate location. This convinces us that we should routinely add shallow boreholes to exploration or production applications where accurate image focus is required.

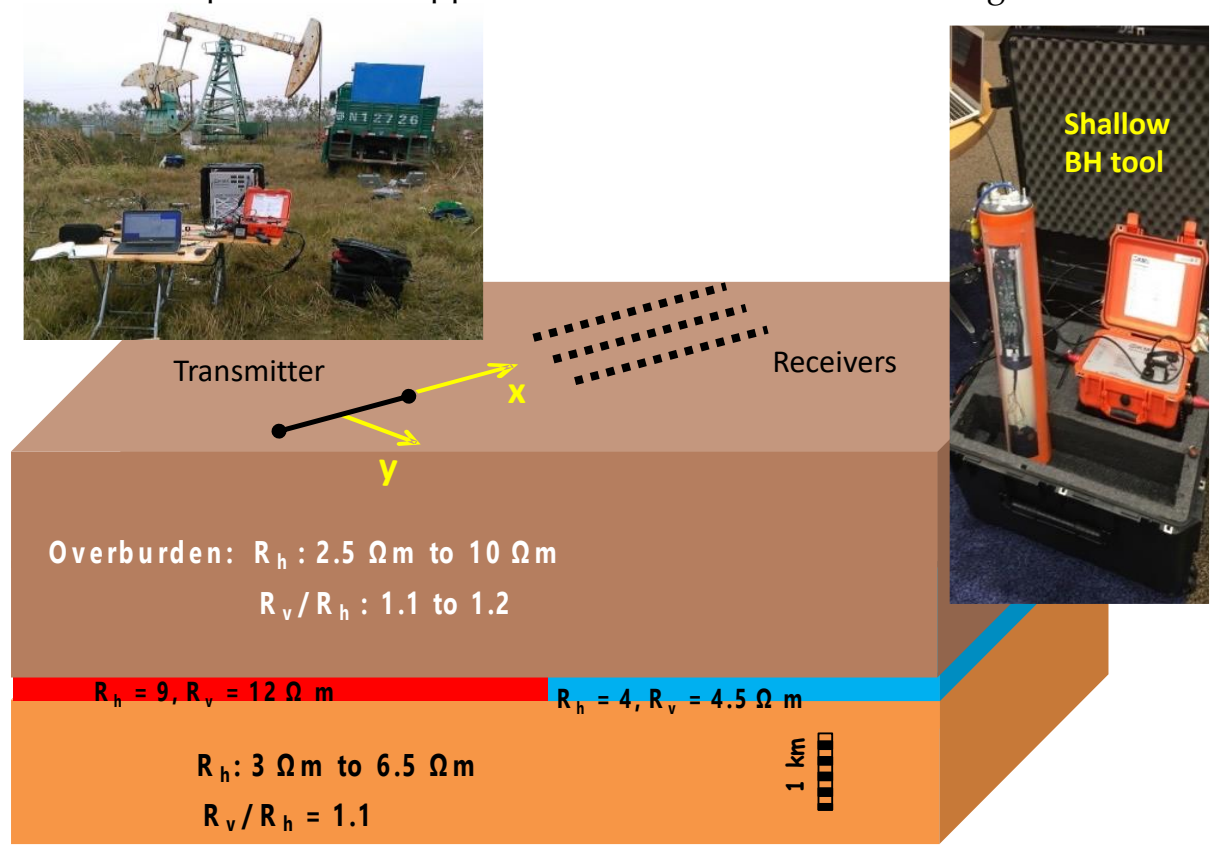

Figure 12. D model showing the anisotropy values derived from well logs for a typical sedimentary reservoir section with the reservoir being just below $2 \mathrm{~km}$ depth. The survey layout of the shallow borehole receiver is shown at the surface. Pictures of the $150 \mathrm{KVA}$ transmitter are at the top left and 


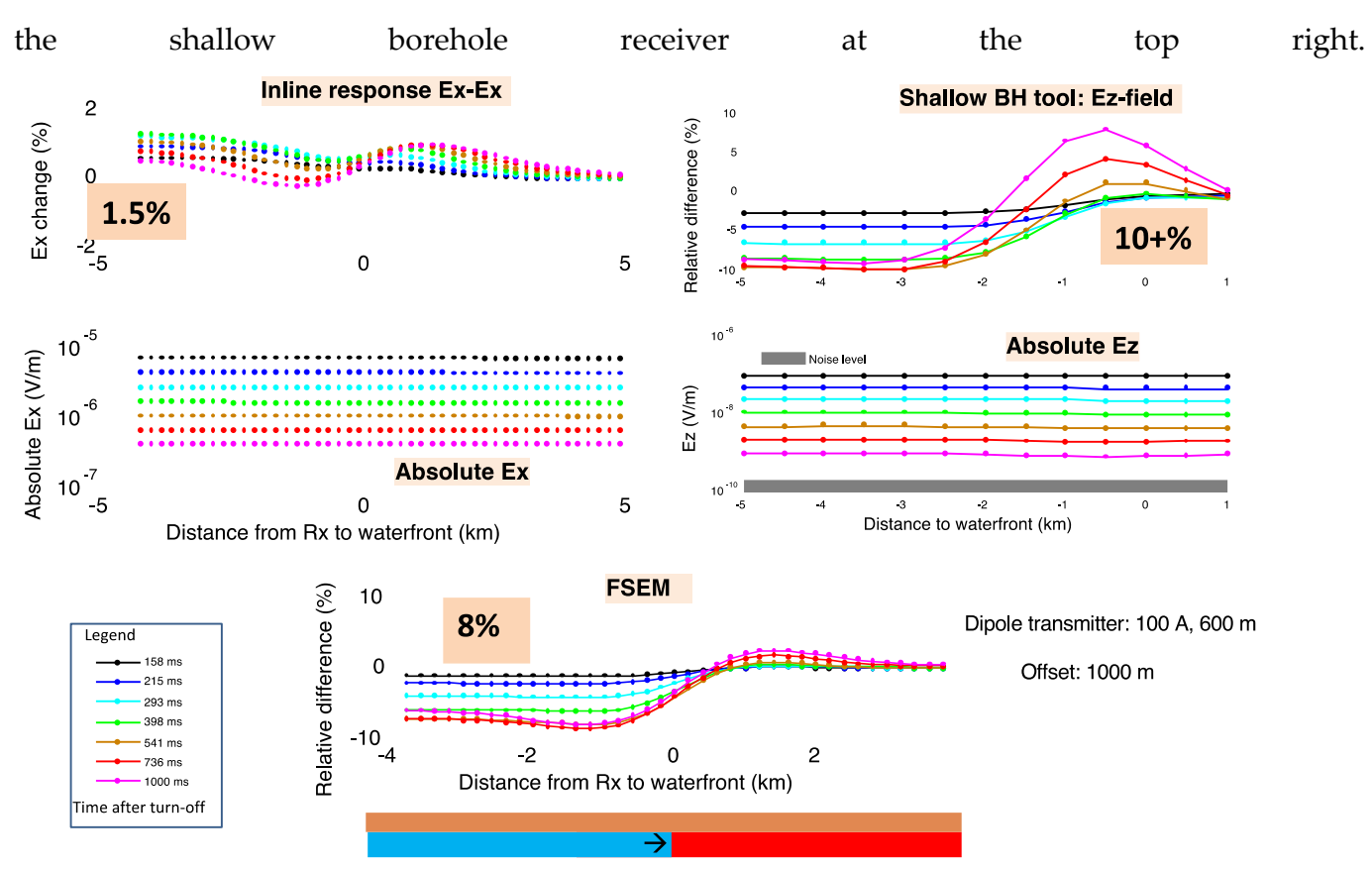

Figure 13. D modeling for the model in Figure 11. At the top are the surface Ex - in line with the transmitter (left) and on the right the Ez response from the shallow borehole tool (right). At the bottom is the FSEM response also measured at the surface. The water flood front is at the center of the profile and the curves are plotted for various times after current turn off.

\section{Reservoir monitoring}

Both land and marine EM monitoring systems include signal generation with a horizontal dipole transmitter and consists of three major parts: surface-to-borehole measurement, surface-to-surface including shallow borehole, and inside borehole measurement. The best solution is to link these measurements in a calibrated manner, while accounting for any information on the anisotropic background resistivity. Coupling EM with seismic provides the additional constraints to better get fluid movements and seal integrity.

It is essential to calibrate time-lapse measurements to the borehole through integrating of surface-to-borehole data. We found over the past 15 years that this is the only way to reliably overcome the inherent equivalence of surface EM measurements. Calibrating borehole against surface EM measurements gives greater sensitivity to fluid variations in the pore space. At the same time linking the EM information to 3D surface and borehole seismic data permits extrapolation away from the well bore and compensation for the loss in layer boundary sensitivity with increasing distance from the EM transmitter. We find it essential to carry out $1 \mathrm{D}$ to 3D feasibility studies for monitoring applications because the reservoir fluid variations will automatically make this a three-dimensional problem. We always include anisotropy from well $\log$ (measured or estimated) and usually surface noise measurements. An example of such studies is given below.

On the hardware side the limitations of EM monitoring have been in the past equipment cost and lack of integration between transmitters and receivers, which allow only a single transmitter and unfocused dipole receivers to be used. Adding today's accurate timing and sequencing to modern hardware we can produce well synchronized transmitters with a current stability better than $0.5 \%$. We can use better arrays that allow volume focusing to increase the spatial resolution and share transmitter and receiver data in real 
time via the Cloud [43]. For marine/borehole applications atomic clocks would be added having accurate time on land and underwater.

Given modern hardware, 3D modeling, and calibration can address the key challenges of CSEM, leaving inversion, integration, and interpretation as remaining challenges in term of producing uncertainty. For example, to reach sufficient depth, one needs to deploy high power transmitter, which brings operations health-safety-environment issues. These issues can all be addressed by careful operation on the grounding and deployments side [55] (see also above). The biggest issue is to use sufficient large and deep electrode plants to avoid building active galvanic cell that dynamically degrade. We thus deploy multiple electrodes on each transmitter side and monitor their performance daily. Careful operations have proven effective even in very dry and hot environment.

The volume focusing issue was discussed above. We can address it using two approaches: FSEM and shallow borehole measurements. For FSEM first successful field test with this technique has been carried out on land [38], and further enhancement of the spatial resolution was reached through integration and joint interpretation with seismic $[13,39]$. We carried out more tests over a salt dome with some initial results shown in Figure 11. For the shallow borehole tool field testing is still in progress.

We use our technology for high value problem such as reservoir monitoring or shale applications. We are at the time where hardware, methodology, interpretation tools and integration progressed sufficiently to rethink the use of controlled source EM on land and offshore. For land application both transmitter and receiver were redesigned to reach a quality standard that allowed high fidelity operation with the current injection at the transmitter. The receivers work reliably all over the world in arid as well as wet and frozen environment. On the receiver side we learned that seismic architecture is not sufficiently comparable with EM architecture because seismic signals are band limited (within a fraction of a decade and EM signal are broadband (DC to at least $10 \mathrm{kHz}$ ). While you can use EM acquisition units for seismic using seismic for EM acquisition usually distorts the data. Today, with real time connection via the Cloud [43] we can also move to the next stage of improving the uncertainty of operations, processing, and interpretation by adding artificial intelligence as described in [43].

When applying EM for reservoir monitoring a proper Feasibility including 3D modeling of the target and integration of all additional information is essential to control operational survey parameters. Usually, seismic horizons, geologic background, and detailed resistivity logs are used as a priori information. Estimating the noise by measuring it with a variety of sensors in the survey area helps to decide if we can resolve the target variation by allowing us to optimize acquisition parameters such as sensor type, sampling rate and acquisition time. When we used this approach during the past 30 years, we have always been able to reach the survey objective with great success. Some of the results from the 3D feasibility modeling directly define acquisition quality parameters. Figure 14 . shows a summary of such a feasibility workflow. A typical feasibility workflow for monitoring applications is on the top left. While it is best to have a 3D induction log, it is not always available and then we estimate anisotropy. We use equivalencing for cumulative transverse resistance and cumulative total conduction to get the end members of the vertical and horizontal resistivities [54,55]. Different 3D modeling codes can give different responses to different models including artifacts, so benchmarking the codes for the target model is essential. This allows you to distinguish modeling artifacts (like over/undershoot - caused by numerical approximation errors) from real anomalies caused by real 3D geologic targets. The high resistivity contrast causes charge accumulation at its boundaries, which is wanted anomaly but also can be mistaken as numerical artifact. Fluid substitution using Archie's formula is sufficient unless you work with shale reservoir where you need to account for an anisotropic saturation Archie's equation. On the right side of the figure, the final composite result of such a feasibility is shown. This reservoir has 
mostly marine sediments with very low resistivities. Its electrical anisotropy is also low. Accurate 3D seismic was given and seismic horizons were used to constrain the reservoir top boundary. Given the surface situation (farmland, villages, pipelines \& power lines), a surface line was placed with color coded receivers corresponding to the curves on its right. Target variations resulted in two different sets of 3D modeling results for different resistivities representing depletion of the reservoir. The corresponding curves are shown in the figure as solid and dashed lines, respectively. Note the curves vary over 4 decades in amplitude (c.f. above comment on amplifiers). We took the noise measurements during an ordinary day with normal EM noise from nearby village, farming and factories. The noise floor of each specific sensor is displayed as horizontal line. Here, we used a standard induction coil, a transient EM optimized induction coil and two air loops with different equivalent areas. The lowest noise floor and largest signal range we obtained with the larger air loop. This was no surprise, since during the exploration for this geothermal reservoir over 40 years ago, it was defined using SQUID magnetometer that's about 1 million times more sensitive than the best of these sensors. In addition, we tested various sampling rates and recording time given the specific noise in this area. Here, the objective was to monitor production in an active geothermal field.

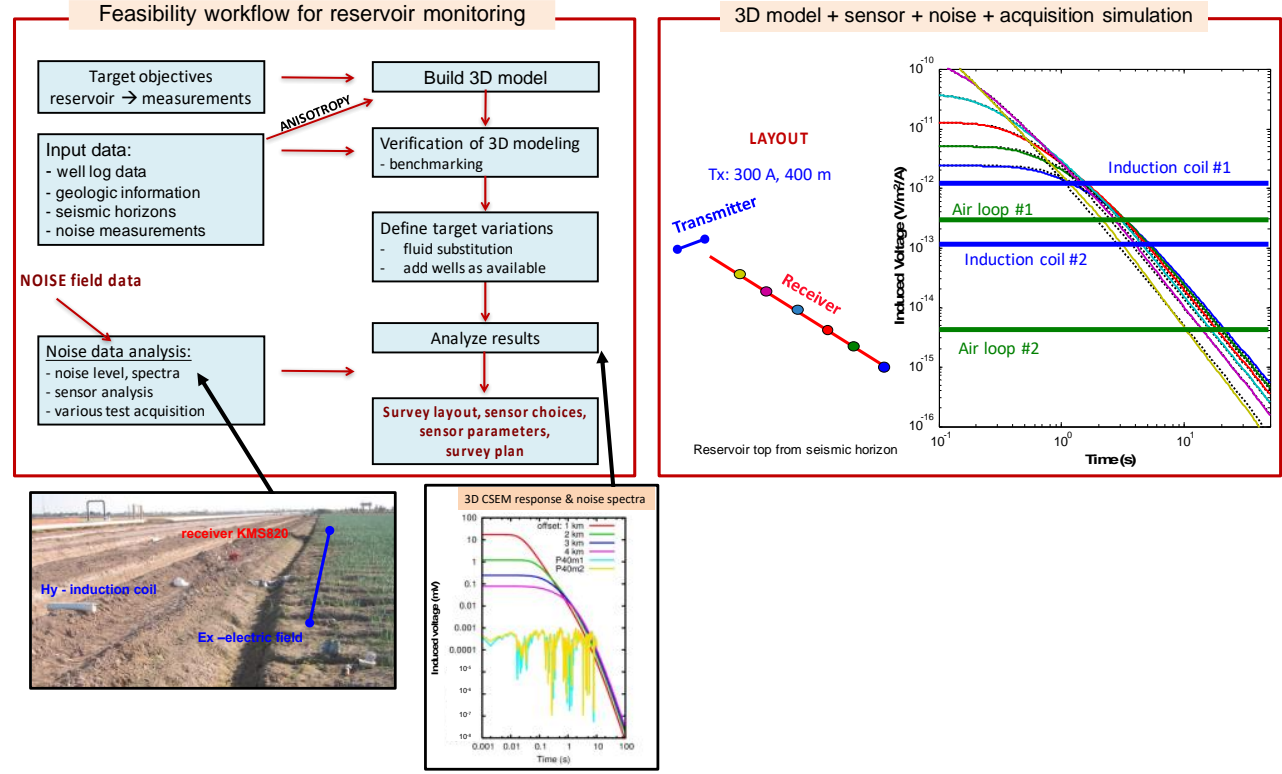

Figure 14. Summary of a typical feasibility workflow and results including 3D modeling combined with noise measured in the field for various sensors simulation reservoir production. On the top left is the workflow starting with a priori information and leading to survey design parameters. Belo is a picture of the sensor sin the field during the noise test and on its right a sample of the various noise spectra compared with the various modeling scenarios. On the top right is the composite result which includes on the left a potential survey layout and to its right the 3D modeling responses for different saturation scenarios (hot or cold fluids). Superimposed are the noise floors for the various sensors.

The next example in Figure 15 is from a water flood in a production oil field with a target at approximately $2.5 \mathrm{~km}$ depth. We derived an anisotropic model from the logs, carried out 3D feasibility modeling and measured the noise at the site. We then build a specific system base done the results and set up a field test. While the absolute resistivities are specific to this oil field, looking at the resistivity ratio, this could represent a producing field in many countries (see Figure 10). We selected some representative monitoring results for two of the sites and displays time lapse differences for a period of 3-5 days after water injection (test data). Since water is more conductive than oil, we used the vertical magnetic field component on the surface, typically more sensitive to conductors. On the left side there are the received voltages and on the right the time lapse differences. The top row represents the receiver directly above the water flood and the row below a receiver $300 \mathrm{~m}$ away from the flood front. Directly above the waterfront the time-lapse 
difference is about $30 \%$ and in the far away receiver we can still see $2 \%$. Since these are test data and relatively noisy, there was still significant noise and thus processing required to obtain reliable stable results. Thus, we are displaying in the vertical line the filter cut off. the left the data are influence by the processing. Also displayed are the time window where we see the target reservoir changes. This field would have been the right candidate to use the above suggested FSEM and shallow borehole measurements. Only using a very stable and accurately current controlled transmitter and long-term stable receivers allowed this. Further survey design like this for heavy oil reservoir monitoring can be found in [21].

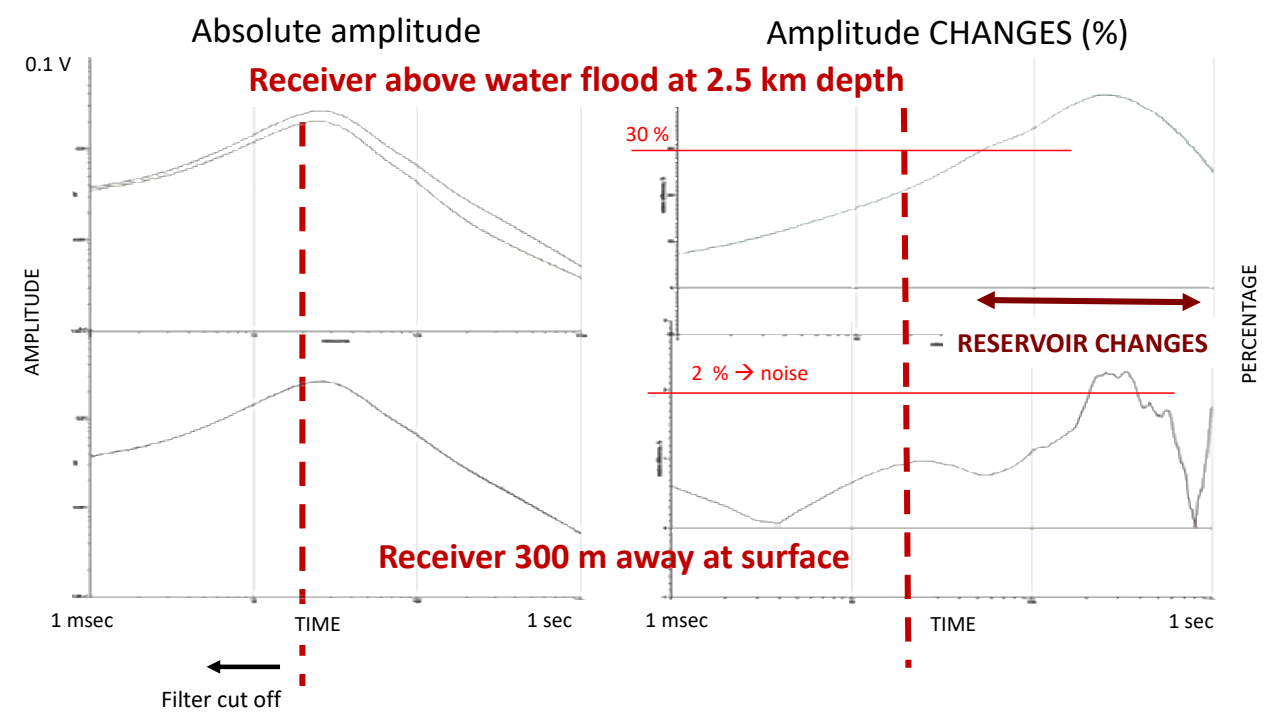

Figure 15. Time lapse difference for an oil reservoir under water flood. On the left are two timelapse responses for the receiver above the injection point and about $300 \mathrm{~m}$ (at surface) away from the flood front. On the right are the percentage time-lapse differences.

Above we introduced a shallow borehole measurement to get better image focus and stronger anomalies. Here, we will integrate deep borehole measurements because on a producing oil field such wells always exist, and the value of the measurements is high enough to afford these. Since oil field environment are often associated with explosive borehole fluid, we prefer using receivers in the borehole instead of a transmitter with high current and voltages. This also allows us to integrate better with surface hardware and we can use transmitters that are 100 to 1000 times stronger than in the borehole. It also has the advantage to get the receivers away from the surface EM noise, which is mostly at the surface. One more item to consider is that in a typical oil well only a small part (the reservoir zone) has no casing, so we need to consider our ability to measure through steel casing.

To illustrate the response of the different measurements we use the Bakken reservoir, which is around $3000 \mathrm{~m}$ depth. The Bakken system covers parts of North Dakota and Montana in addition to parts of Saskatchewan and Manitoba, Canada, and includes the Bakken, Lower Lodgepole and Upper Three Forks Formations. A geologic model and the array setup with the well log are shown in Figure 16. The Bakken Formation is comprised of three distinct members, the upper and lower Bakken's organic rich shale layers, and the middle Bakken member, which is primarily sandstone and siltstone. The middle Bakken is the primary reservoir rock [67] but production has been extended to the other formations. Our objective is to monitor depletion of the hydrocarbon. In term of EM modeling this is like water flood monitoring, and we are looking for a target with increasing conductivity. The receivers are in the horizontal part of the top well in the Middle Bakken and at the surface. The transmitter is at the surface along the $\mathrm{x}$-axis and the coordinate system origin for the plots is in its center. 


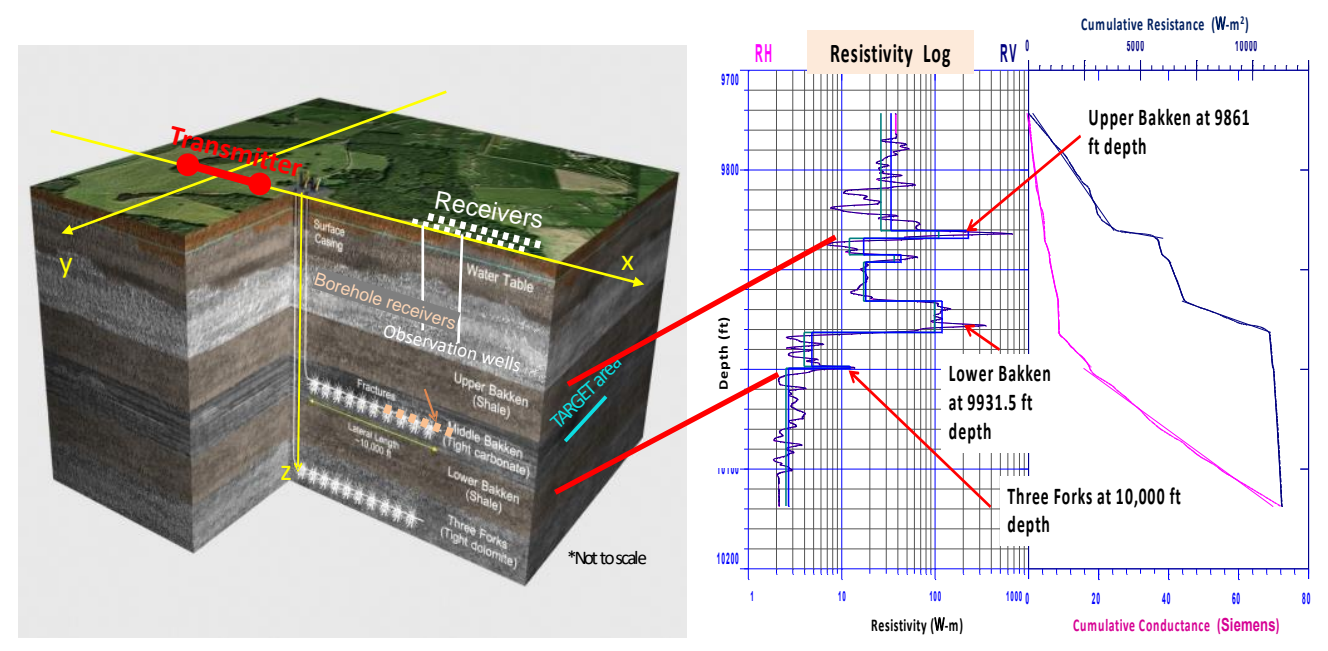

Figure 16. Model and survey setup and model for the Bakken reservoir in North Dakota. The receivers are placed in horizontal wells and the transmitter is at the surface as shown. On the right is the resistivity log and the resulting anisotropic model derived from it, using the cumulative conductance and cumulative transverse resistance calculated directly from the log and interpolated as shown.

Figure 17. shows 3D modeling results demonstrating good sensitivity of time-domain measurements to a waterfront moving from negative y-direction from a parallel injector well situated inside the reservoir at the same depth as the producer well (see Figure 14.). The target area for depletion monitoring is on the cross-section as shown in light blue. The borehole receivers are situated at $\mathrm{x}=1000$ to $4000 \mathrm{~m}$ inside the lower Bakken reservoir in $(x, z)$ plane. The waterfront was modeled as a rectangular block of vertical extend of $31 \mathrm{~m}$, the horizontal extends of $4000 \mathrm{~m}$ in $\mathrm{x}$ and $400 \mathrm{~m}$ in $\mathrm{y}$. The resistivity of the flooded area $(8.16 \Omega \mathrm{m})$ was derived using Archie's law considering the reservoir porosity of $7 \%$ (courtesy of Microseismic Inc.). The background 1D (horizontally layered) anisotropic resistivity model was derived from a vertical log as shown in Figure 16. on the right. Figure 17 shows synthetic responses of the borehole magnetic receivers as a function of their location (left) or as a function of time after turn-off. Since the background model is symmetric with respect to $(x, z)$ plane, $B y$, is the only non-zero magnetic component in the borehole receivers inside the oil reservoir before the production start. Therefore, $\mathrm{Bx}$ and $\mathrm{Bz}$ are equal to zero and not shown. As the oil gets depleted and the anomalous (light blue) zone approaches the producer well, a non-zero Bx and Bz emerge, which can be analyzed to determine the distance to the waterfront. Further applications include monitoring hydraulic fracturing which is one of the next frontiers, and EM is surprisingly effective in this [68]. Note that the electric field shows mostly early-time anomaly, while the magnetic field always displays anomalous response. 

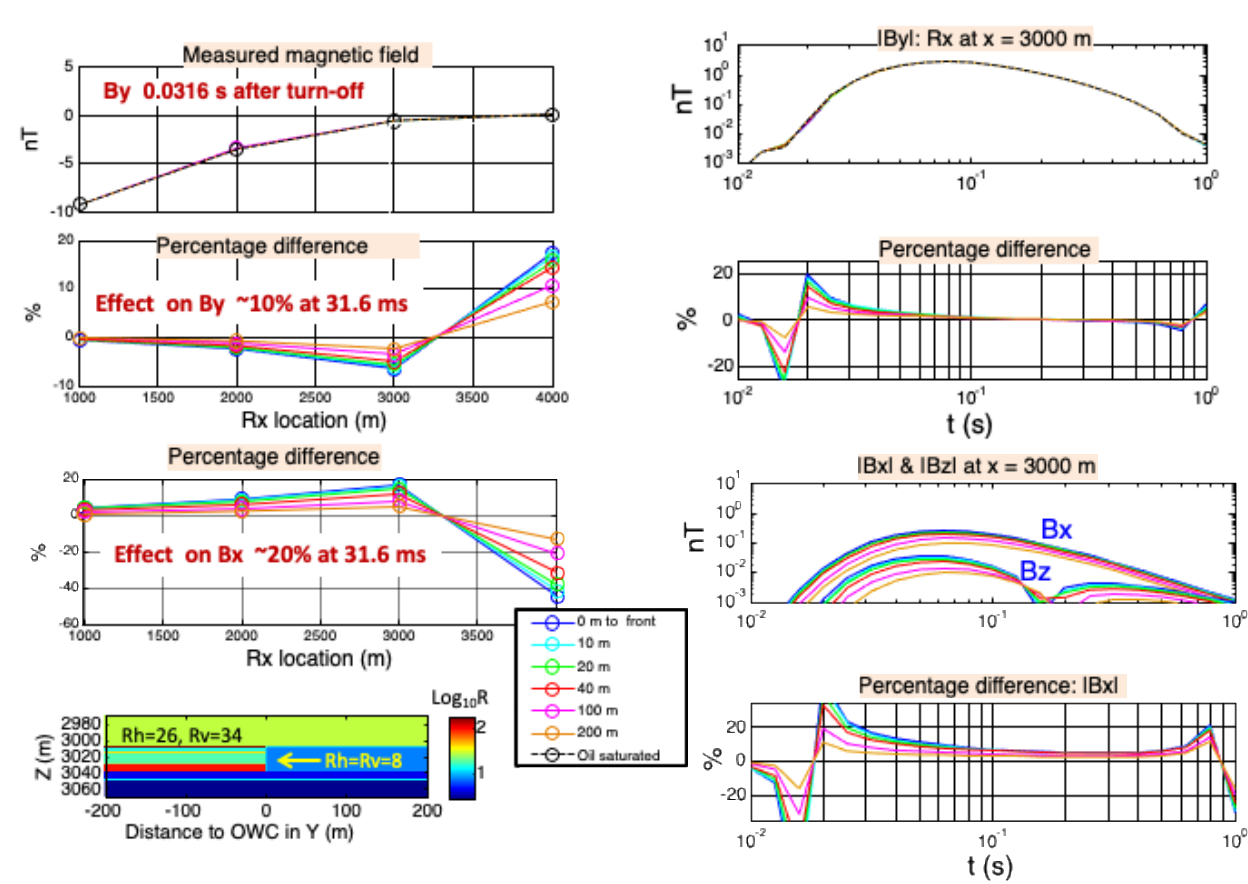

Figure 17: Modeling (3D) results for the Bakken model. We selected the magnetic field, By, as it is nonzero. By and its time derivative are shown at the top, and the percentage variation due to depletion (simulating the distance to a sharp Oil-Water-Contact - OWC) is used. The percentage change in Bx while initially smaller gets larger than $\mathrm{Bz}$ as it gets closer to the OWC. The resistivity model is shown on the right. The transmitter is at the surface and the receivers at $3000 \mathrm{~m}$ depth.

The 3D modeling results in Figure 17 demonstrate good sensitivity of time-domain measurements to a waterfront moving from negative y-direction (measured from a parallel injector well situated inside the reservoir at the same depth as the producer well as in the model in Figure 15.). The target area for depletion monitoring is on the cross-section as shown in light blue. The borehole receivers are situated at $x=1000$ to $4000 \mathrm{~m}$ inside the lower Bakken reservoir in $(x, z)$ plane. The waterfront was modeled as a rectangular block of vertical extend of $31 \mathrm{~m}$, the horizontal extends of $4000 \mathrm{~m}$ in $\mathrm{x}$ and $400 \mathrm{~m}$ in $\mathrm{y}$. The resistivity of the flooded area $(8.16 \Omega \mathrm{m})$ was derived using Archie's law considering the reservoir porosity of 7\% (courtesy of Microseismic Inc.). The background 1D (horizontally layered) anisotropic resistivity model was derived from a vertical log as shown in Figure 15. on the right. Figure 17 shows synthetic responses of the borehole magnetic receivers as a function of their location or as a function of time after turn-off. Since the background model is symmetric with respect to $(x, z)$ plane, by is the only non-zero component of the magnetic induction in the borehole receivers inside the oil reservoir before the production start; it is why $\mathrm{Bx}$ and $\mathrm{Bz}$ are equal to zero and not shown. As the oil gets depleted and the anomalous (light blue) zone approaches the producer well, a non-zero $\mathrm{Bx}$ and Bz emerge, which can be analyzed to determine the distance to the waterfront. Further applications include monitoring hydraulic fracturing which is one of the next frontiers, and EM is surprisingly effective in this (Palish et al. 2017). Note that the electric field shows mostly early-time anomaly, while the magnetic field always displays anomalous response. Clearly, the magnetic field can see the approaching waterfront which makes this an invaluable tool.

Borehole measurements with the electric field sensors are uncritical. As magnetic field sensor we have selected the same fluxgate sensor that we use for MT and the shallow borehole tool. Its sensitivity curve is shown in Figure 5. The sensors were originally downsized for a marine system. It includes all electronics at the sensors with 32-bit digitization 
and part of the first series production. From Figure 5, for LEMI-029 and LEMI-024 fluxgate sensors, we can see that the frequency used in Figure $18(0.1111 \mathrm{~Hz})$ has a noise of 5 pico-Tesla while the signals in Figure 18 are in the mili-Tesla range. This means that the existing sensors can be used for measuring of the magnetic fields inside of the steel casings.

Obviously, once you are in the wellbore, you can carry more EM measurements through casing and in open hole to achieve an even higher degree of integration. To model realistic steel casing environment using correct and representative casing parameters is paramount. We used the casing parameters (thickness, conductivity, permeability, and temperature variation) used for a real through casing resistivity logging tool design with 3D modeling [69] to get realistic responses. These results were subsequently confirmed by measurement and finally by independent tests with several logging tools from different manufacturers [70]. We thus feel confident that these are realistic. The survey configuration is the same as in Figure 15, with a surface transmitter, surface receiver and electric and magnetic field receivers inside the horizontal well section. To avoid surprises due to numerical inaccuracies we used 3D finite element (FE) and 3D finite difference (FD) modeling programs. We are now looking at the data in the frequency domain since we know casing effect can be best dealt with at frequencies below $0.5 \mathrm{~Hz}$ [69] to avoid/reduce skin effect in steel. Figure 18 also shows the results for the inline electric field Ex and the downhole vertical electric field, Ez, and horizontal magnetic field. We are looking at booth imaginary a, Im, and real parts, Re, quantities. The finite element modeling results are the solid lines and the finite difference the symbols. The 3D modeling responses with metal casing are in black and the 3D responses without casing are in red, pink, and green respectively. Except in the upper $500 \mathrm{~m}$ for the vertical electric field (center plot in Figure 17.) the casing effect is small. As expected from around $1 \mathrm{~Hz}$ and higher frequencies these effects are increasing (not shown here), which is consistent with [69]. The sensor used for the magnetic field are the same fluxgate magnetometers with 4 pT resolution (LEMI-029 shown in Figure 5), which will give us no resolution issues since the magnetic fields are in the micro-Tesla range as shown in Figure 18.

This is excellent news as it means we can build a through casing surface-to-borehole system with existing sensor components.

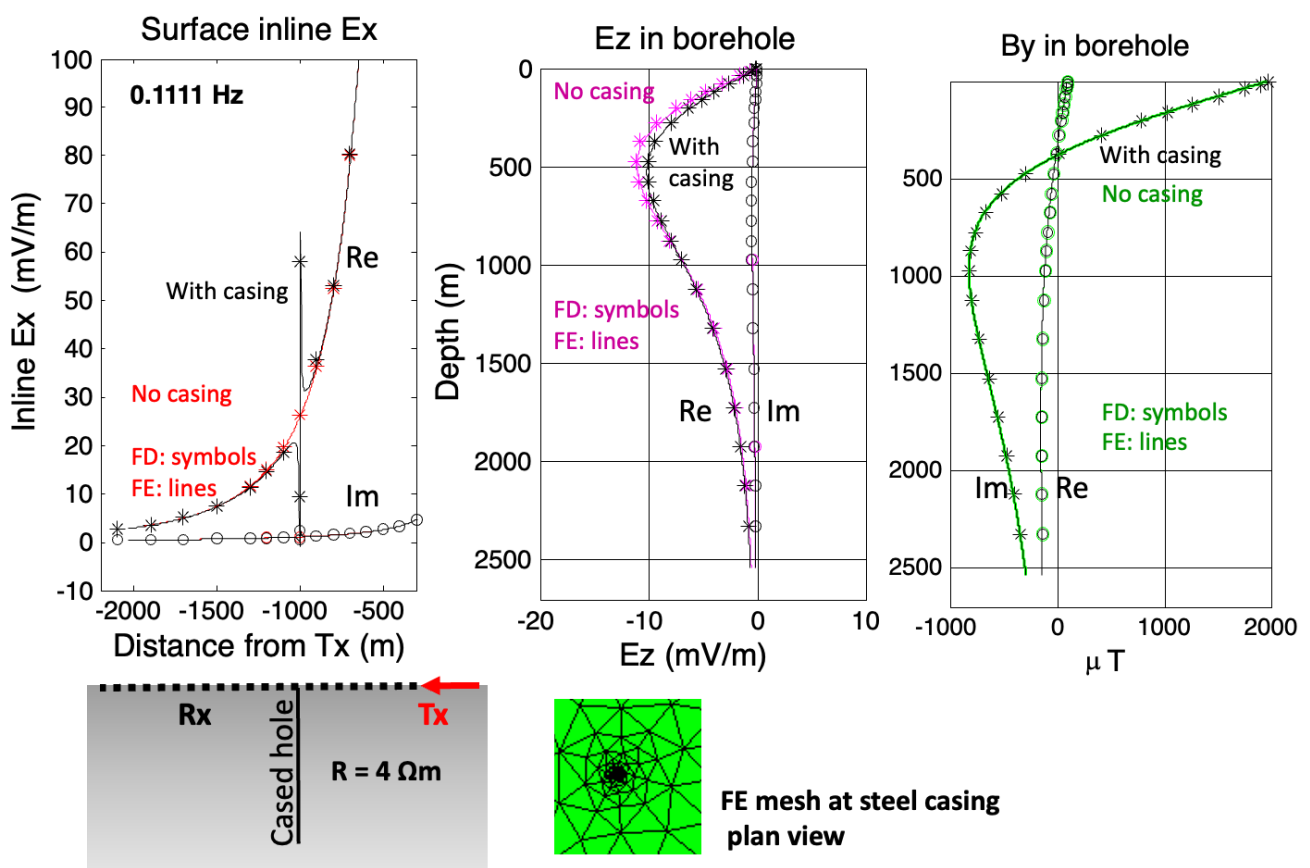


Figure 18. D modeling results using finite element and finite difference formulations to model the influence of casing effect to surface-to-surface and surface-to-borehole measurements. On the left is the inline electric field with the origin being at the transmitter. A layout is shown below. The center plots show the vertical electric field and on the right is the magnetic field in y-direction (with respect to the transmitter being in $\mathrm{x}$-direction). Real and imaginary parts are shown for a frequency of 0.1111 Hz. Superimposed are results with and without steel casing.

\section{Resulting requirements}

The applications for these methods build the superset of all industrial requirements, while we are focused on high value future application of reservoir monitoring. Since we depend on increasing acceptance of EM, we include standard explorations applications to continuously improve hardware and system concepts. The overall objective is to bridge the gap between EM and seismic as given on land by operational requirements establish by the industry over the past 75 years.

Most of the survey cost lies in the logistics and crew operations (90\%). If you can operate EM together with seismic you can reduce the cost. To use the same crew, system operations must be similar in simplicity and only minimum training must be required for system deployment. This leads to nodes with almost automatic deployment. Long operating times require low power consumption. Transmitter and receiver operate autonomously.

Seismic images derive their strength from data redundancy. Thus, EM system must be scalable and modular to be able to reduce cost in a fit-for-purpose fashion leading to a variety of sensor options with optimized cost/sensitivity. Each node can be extended by either wired or wireless sub-acquisition controller to principally unlimited channel numbers and allow an overall reduction of cost while individual nodes are still expensive.

Wireless low weight system for helicopter (airborne) operations in complex terrain as well as nodes with wired sub-acquisition controllers (to reduce cost further) for dense 3D applications. Power consumption must be low, and GPS must be integrated in the unit.

Special operations must be possible like: Buried or underground receivers, and transition zone recording requiring handshake between GPS and internal timing. For dense vegetation external GPS antenna or better internal timing like atomic clocks should be an option.

The internal timing to the system extensions for borehole and marine operations.

Obvious are standard requirements for ruggedness and environmental extremes like heat, cold, resistant to cattle, shockproof, and waterproof.

When designing a system, the specifications should be a result of fulfilling most requirements not just cost and/or weight. In the same fashion the processing specification should result from the requirements. Both set of requirements for hardware and software are to be considered as technology and should be tailored to the target problem on hand.

Processing

Because of all the special variation of EM and its changing sensitivity with depth, every EM method had special ways of acquiring and processing. When seismic processing became more accessible to academia, students and companies around Colorado School of Mines began in the early 1980 to adopt seismic recording standard to EM. Later the processing followed, but EM needed true amplitude processing from the very beginning as the signal must be calibrated. So true amplitude processing was used in EM well before in seismic [71]. After adopting seismic hardware standards, further standardization occurred and interest in the seismic community grew [72]. After hardware and data standards, the processing and interpretation became so transparent that time and frequency domain processing gave almost identical results [73]. We experienced that each target requires different adaptive workflow and because the EM signal is diffusive and its frequency content changes with depth, we must validate our workflow every time. Keeping the processing effects far away from the signal band and at a minimum appears to be the best suggestion (especially for monitoring). 
If the system is designed efficiently, the difference between methods lies only on the data processing side including inversion or time lapse sections. Figure 19 shows the highlevel workflow for processing and integration of EM and microseismic. The EM stands for MT and CSEM for which we have adopted similar workflows. If a node acquires multimethods, the different methods are separated in pre-processing steps to conform with the industry format and standards for that method. After that comes the signal averaging (stacking) and a post-processing step to convert the results into a measurement quantity that represents the subsurface structure. The microseismic and EM data comes back together when we model has been derived (Figure 19 on the right). After the inversion or imaging, the results get integrated again for a unified interpretation and integration. Here, data errors and sensitivities are equally important as the match to existing 'hard' geological information. It has been our experience that the integration and integrated interpretation takes at least as much time as the interpretation of each method alone.

The data separation in repeat surveys (time-lapse) is necessary, because EM data can be affected by a variety of cultural noise. The first step in successful analysis of any EM geophysical methods is proper processing. Routinely, we implement several approaches which insures the most robust results that can be derived from recorded data. With robust statistical procedures we derive stable transfer functions for MT method as well as CSEM. Special care is taken to estimate smooth and stable transients in time domain.

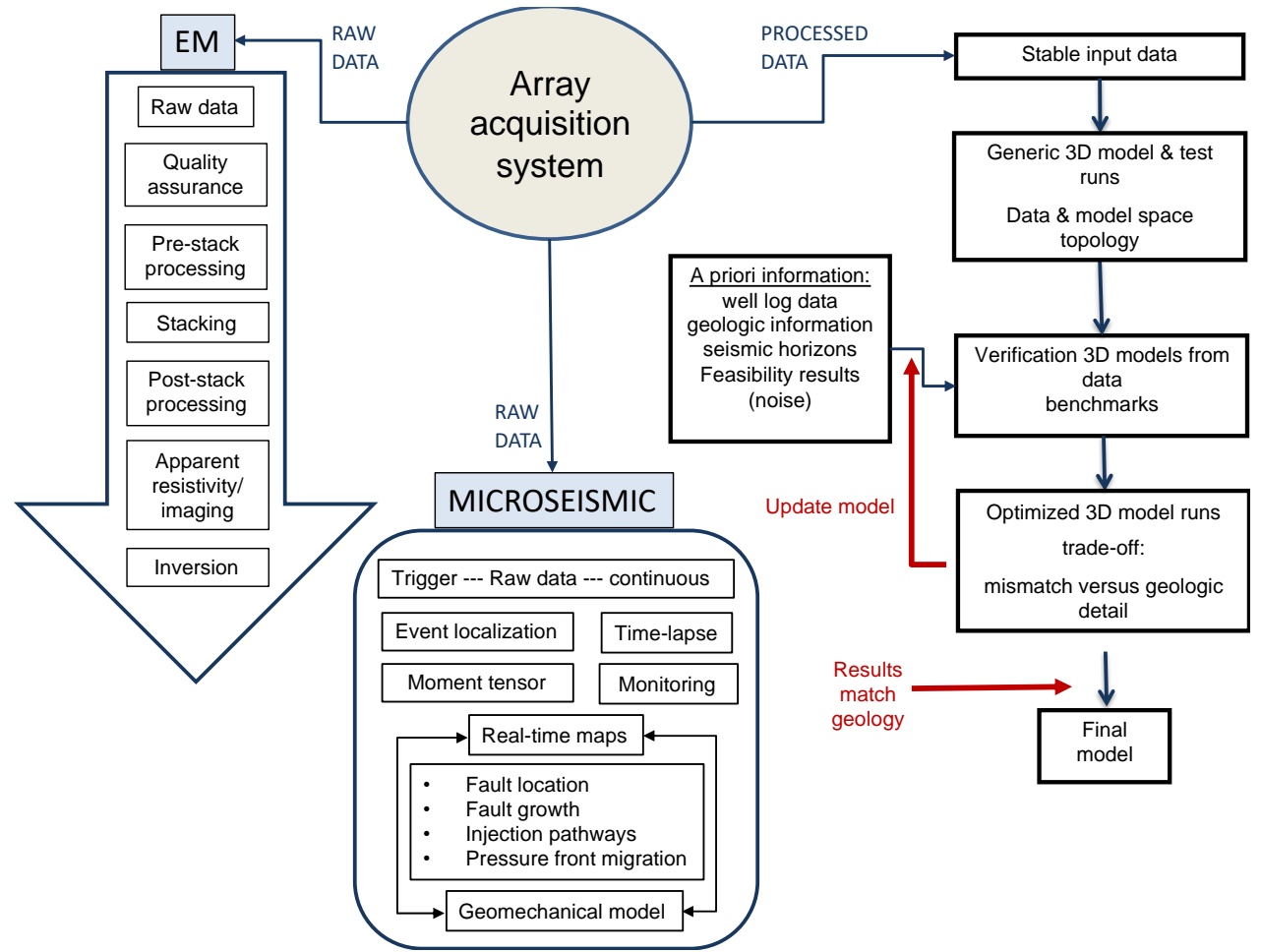

Figure 19. Data processing workflow for MT, CSEM, and microseismic. The data can be acquired separately or simultaneously by the same array system. It is the processes as data set separated by physics and the results combined again for further integration and interpretation (on the right).

\section{MT data processing}

To estimate MT transfer functions, we follow the extended approach to deal with array data, which is referred as robust multi-remote reference [74]. There are numerous distinct features in the algorithm that make it robust against noise contamination. At the first step, spikes and outliers in time domain are removed based on auto-regression prediction (AR) filtering. Thereafter harmonic noise is eliminated in frequency domain. After that adaptive coherence sorting is applied to select time segments with the best signal/noise ratio. It should be noted that local cultural noise may have rather high coherence, which in some circumstances result in erroneous estimates. The following robust regression estimation having highest breakdown point is used to derive final parameter 
estimates. It includes the Siegel estimator [75] which is based on repeated median algorithm with a reduced M-estimator. This robust processing scheme improves data quality and results in high quality estimations of impedance and tipper despite contamination by industrial noise.

A data example is shown in Figure $20[13,76]$. The apparent resistivity curve on the left shows the data using standard classic averaging and the one on its right with robust processing. In this case the MT site was $50 \mathrm{~m}$ apart allowing us to derive a dense image from the 3D inversion. The total depth of the section is about $1500 \mathrm{~m}$. The section is the most stable 2D inversion which is then used for the integration with other geophysical methods and further modified when integrated with seismic, gravity, and geology (see [13] for the complete case history). Figure 21 shows the interpreted and integrated salt dome model on the left and the model with the MT data on the right. For the left image approximately $360 \mathrm{MT}$ site were added to the seismic and gravity data derived image on the right. The results led to vastly improved seismic images and additional successful production well [13]. The authors attribute the success mostly to the dense station spacing and the multi-physics integration. The improved resolution here is derived most because of the data redundancy with station spacing of as close as $50 \mathrm{~m}$.
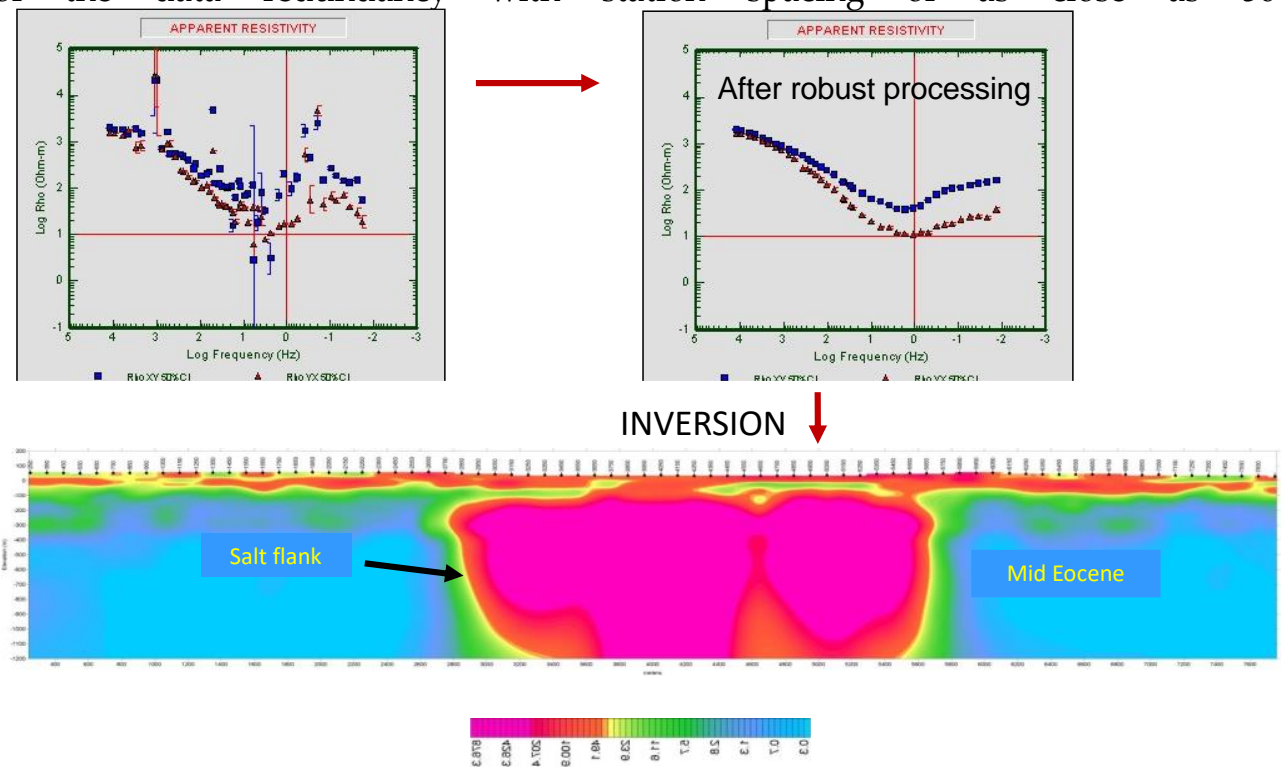

Figure 20. MT data processing example. On the left are the apparent resistivities curves for the twotensor direction $\mathrm{xy}$ and $\mathrm{yx}$ using classic processing and on the right using robust estimator. Both data sets use remote reference sites to reduce the noise. The stable $2 \mathrm{D}$ inversion image resistivity section is shown below. The data is from a salt dome in Northern Germany.

\section{New EM integrated model}

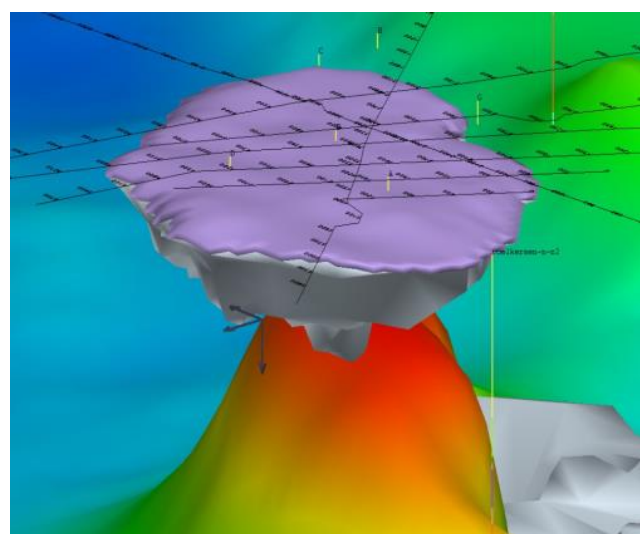

\section{Previous model}

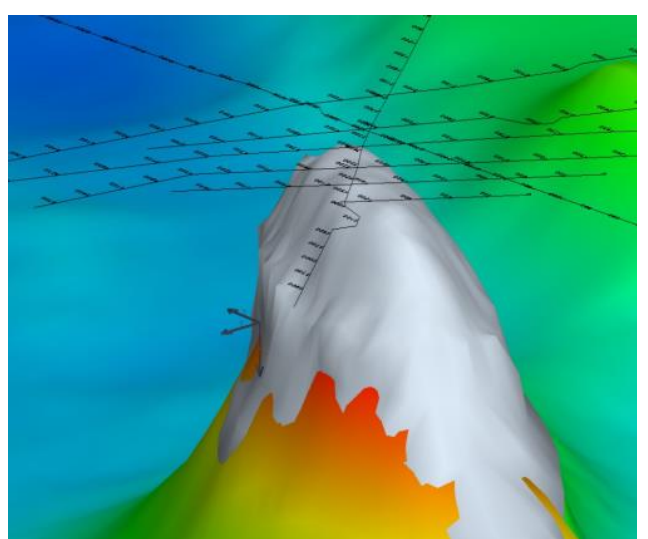


Figure 21. Example of interpreted and integrated composite image including high resolution MT, gravity and seismic (left). We are comparing here the model after the MT measurements with 50 and $100 \mathrm{~m}$ station spacing and without (using gravity and seismic only). The data is from Northern Germany [13].

Benefits of using integrated MT processing [74] with traditional robust processing [77] are the adoption of new statistical procedures, advanced user interface and readily available multi-remote reference processing. The advantages of this style MT processing are:

Processing speed

Predefined processing parameters which are more universally applicable

Optional selective user defined parameters

Multi-remote reference processing

Multiple transfer functions (TF) file robust stacking

Easy graphical handling of multiple impedance or tipper files with different frequency bands

Easy robust merging different frequency bands

Visualization of various MT parameters

Obviously, there are further benefits possible in the industrial implementation of various summarizes basic processing requirements for MT data which are standard for most manufacturers' software.

Table 1. Summary processing functions of a standard generic MT system. Emphasis should be given to data quality and transparency of processing flow as well workflow atomization.

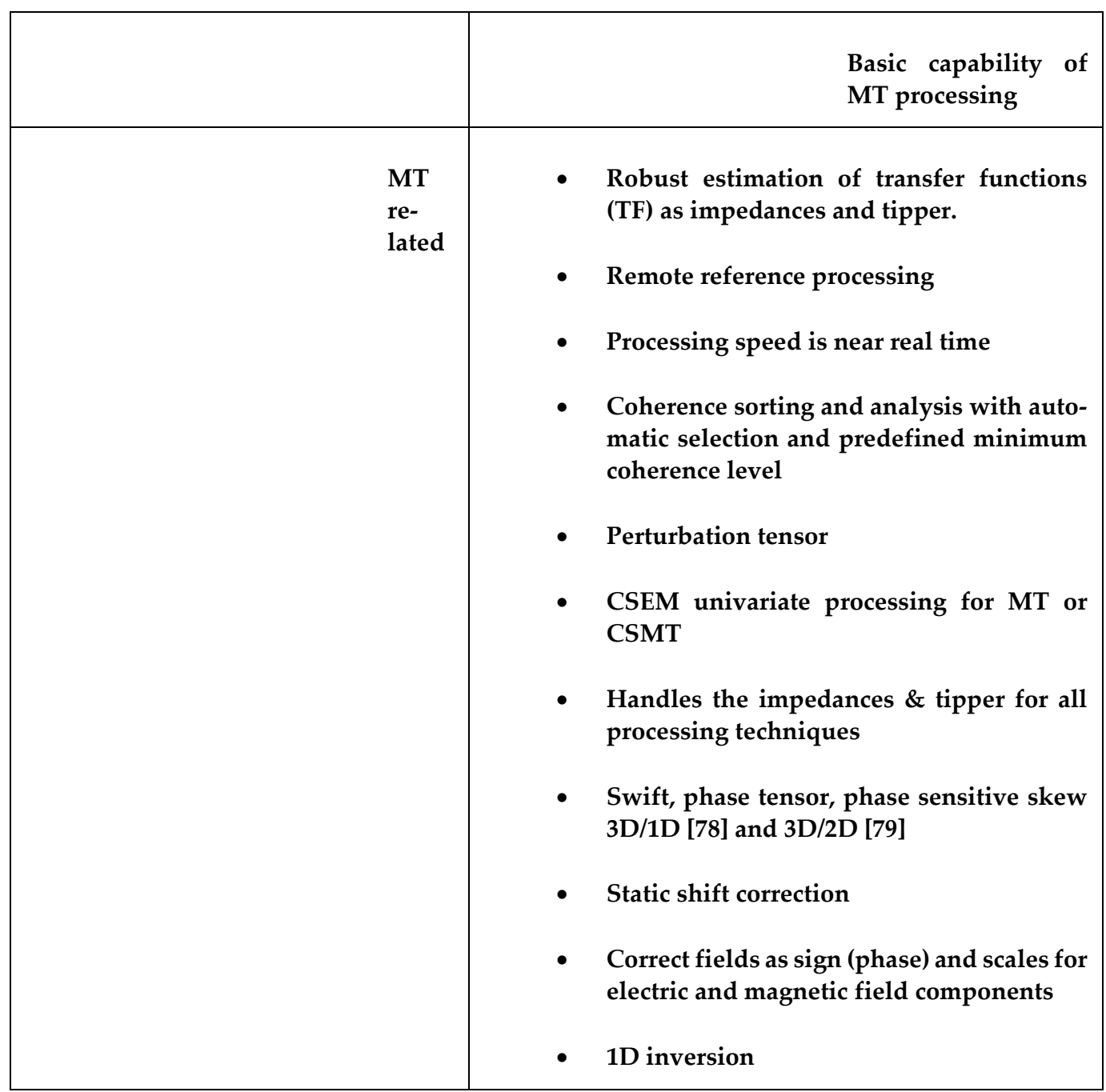




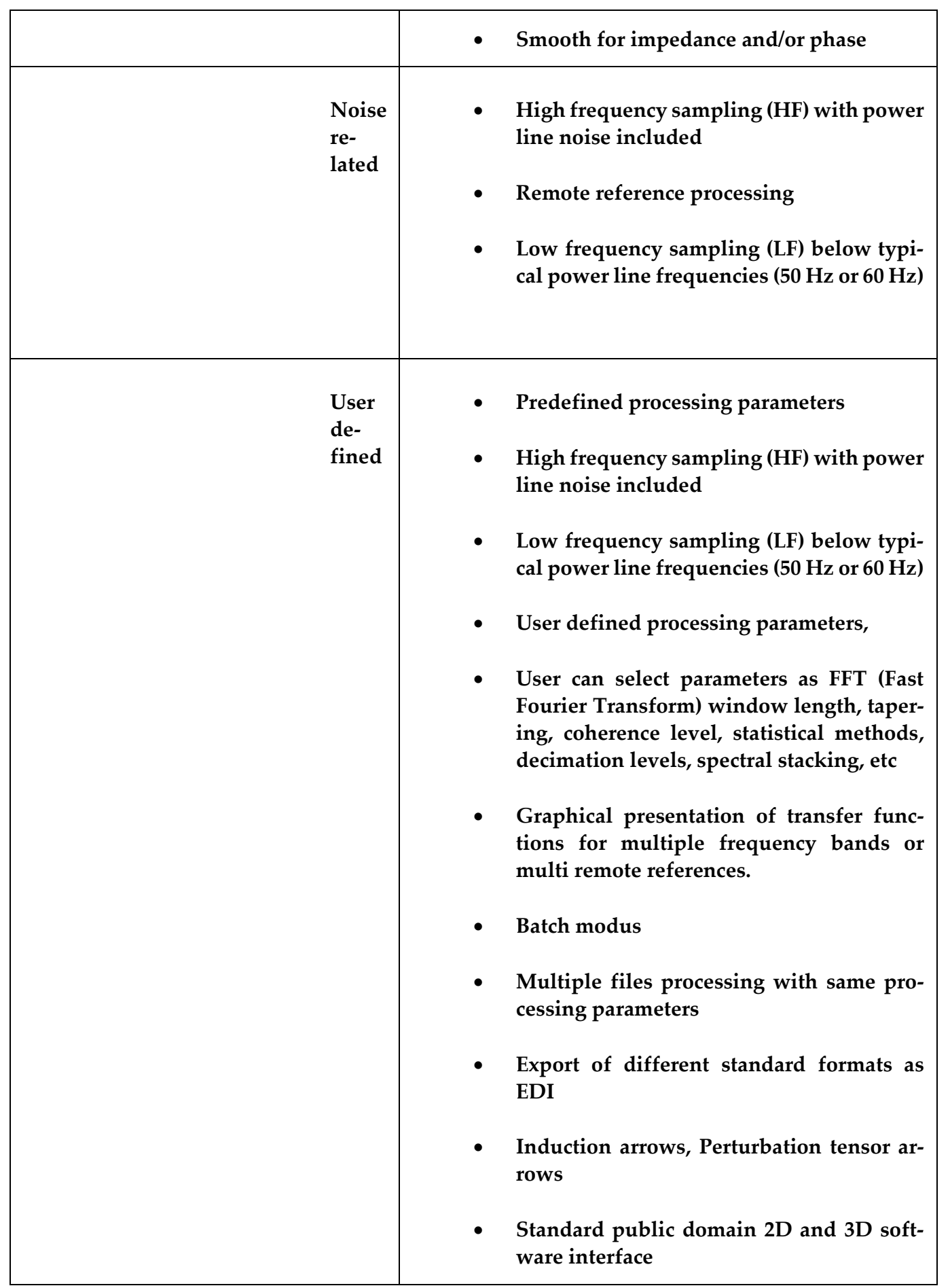

Using the above describe concepts for hardware and processing we acquired data of a 3D structure with the array system and broadband coils. Figure 22 shows the results. The site is $500 \mathrm{~m}$ next to urbanization and an active salt mine and has very high cultural noise. Figure 22 shows data that represent the 3D structure with long recording times. The site is about $300 \mathrm{~m}$ to the West of the Receiver 3 in the 3D model in Figure 10, in the center of the elongated salt structure. The data shows apparent resistivities and phases for both orthogonal directions including error bars. The salt dome is clearly visible in the data. 

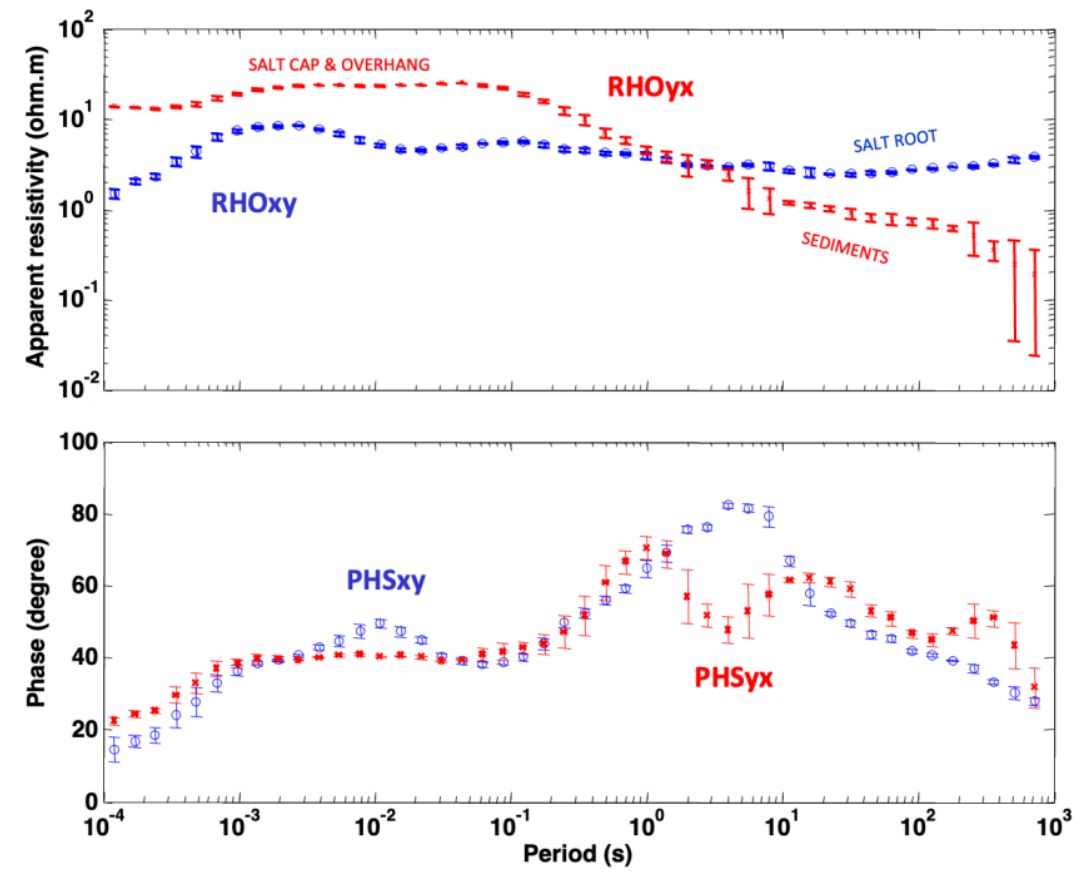

Figure 22. Broadband data set from the Hockley salt dome near Houston, Texas. The data is from 2018, when urbanization made that area very noisy. The data show 7 orders of magnitude in period/frequency above a 3D salt dome (see Figure 10). The yx - component sees initially the salt cap and at longer periods (lower frequencies) samples outside of the salt dome where the conductive sediments prevail. The perpendicular xy-component is still dominated by the salt dome root.

\section{CSEM processing}

CSEM soundings in the frequency domain are usually generated at fundamental frequency with its harmonics. This allows to cover a wide frequency range using one transmitter run, while multiple frequencies are generated at the same time. For time domain soundings only, a square wave is used and the decay curves after the switching are analyzed. For both domains, the data recordings consist of EM field components recorded at various positions on the ground (possibly underground and in the air). At the same time current recordings in the transmitting antenna are performed to exactly evaluate transmitting dipole moment and subsequently used to normalize the received voltages. We have addressed some issues with the transmitter above for operational and processing viewpoint. Noise related to the interaction of transmitter and receiver can be caused by leaking of transmitter signal into power grid infrastructure and/or pipeline network or via metal underground structures and conductive river channels in the subsurface. Both are difficult to correct. For sedimentary basin, CSEM (or Lotem) has been used to investigate the depth range from $800 \mathrm{~m}$ to $3000 \mathrm{~m}$, so we will show a more recent complex example from the same Hockley salt dome (see Figure 10) and then some reservoir monitoring processing applications. Details of the standard processing of CSEM data can be found in $[55,80]$. Lotem which originally referred to with Long Offset (incorrectly), refers today to CSEM with grounded dipole as the offset no longer plays a role with depth of investigation when using today acquisition technology. The clarification of this misunderstanding was the results of a study of the depth of investigation [81].

While the 1980s, most time domain CSEM processing was tailored for the specific time variant nature of the transient signal [55], we now can use either frequency or time domain filters and obtain comparable results. frequencies are well defined in CSEM soundings. Thus, it is possible to perform the Fourier transform at selected frequencies, however the signal to noise ratio is not always high for every frequency as well as there exists the spectral power leakage to the neighboring frequencies. We usually select a targeted spectral resolution to hit transmitted frequencies, but also makes it possible to select frequencies based on the coherence sorting in an efficient manner in a similar manner as 
it is done during MT data processing. After benchmarking the processing with the data sets on hand, we go back and minimize the processing to avoid data error increase caused by processing.

After splitting the entire time series into segments and Fourier transforming them, neighboring frequencies are used to estimate coherence. It also defines the initial spectral smoothing. It is also possible to avoid smoothing the frequencies, but then there will be only one coherence for the whole segment estimated. Only segments having coherence above predefined threshold will be considered. If spectral smoothing was applied, individual frequencies will be examined for coherent signal. We use a variety of filters customized for the method chosen. We find both time and frequency domain filters for, here, time domain data equally useful. Figure 23 gives an example for a typical processing sequence as used for the CSEM measurements around the Hockley salt dome also used in Figure 10. Note, while we used the Hockley area for MT test measurements over the past ten years, closing-in housing projects and a growing salt mine makes this today near impossible. Presently, the effect on CSEM (Lotem) measurements seems to be manageable at this site. In Figure 23 we have the raw magnetic and electric field data at the top left with the averaged (stacked) data sets on the right. Displayed on the top right are a filtered and unfiltered stacked data set. Below are the one-dimensional inversion results display as spliced section or 3D visualized. From the Eigenparameter analysis we can see that the base of the salt overhang and the conductance of the sediments below the salt are resolved. This interpretation is consistent with the 3D model in Figure 11.

Figure 24. shows example of monitoring data of various data quality. The left of the figure shows electric and magnetic field data logarithmically displayed to show the data smoothness at later time. The top data (left side) set uses a $100 \mathrm{KVA}$ transmitter but there are still noise issues remaining. The bottom data uses a $150 \mathrm{KVA}$ transmitter and the data has much better data quality. This is confirmed by using very weak prestack filtering and merely achieving this data quality through stacking. On the right, the data validity is derived by comparing the data with the 3D anisotropic model derived from careful log interpretation and 3D modeling. Note the model resulting from the inversion shows only small variation from the $3 \mathrm{D}$ model. This means the data is representation for the geology and the variation represent local site variations.

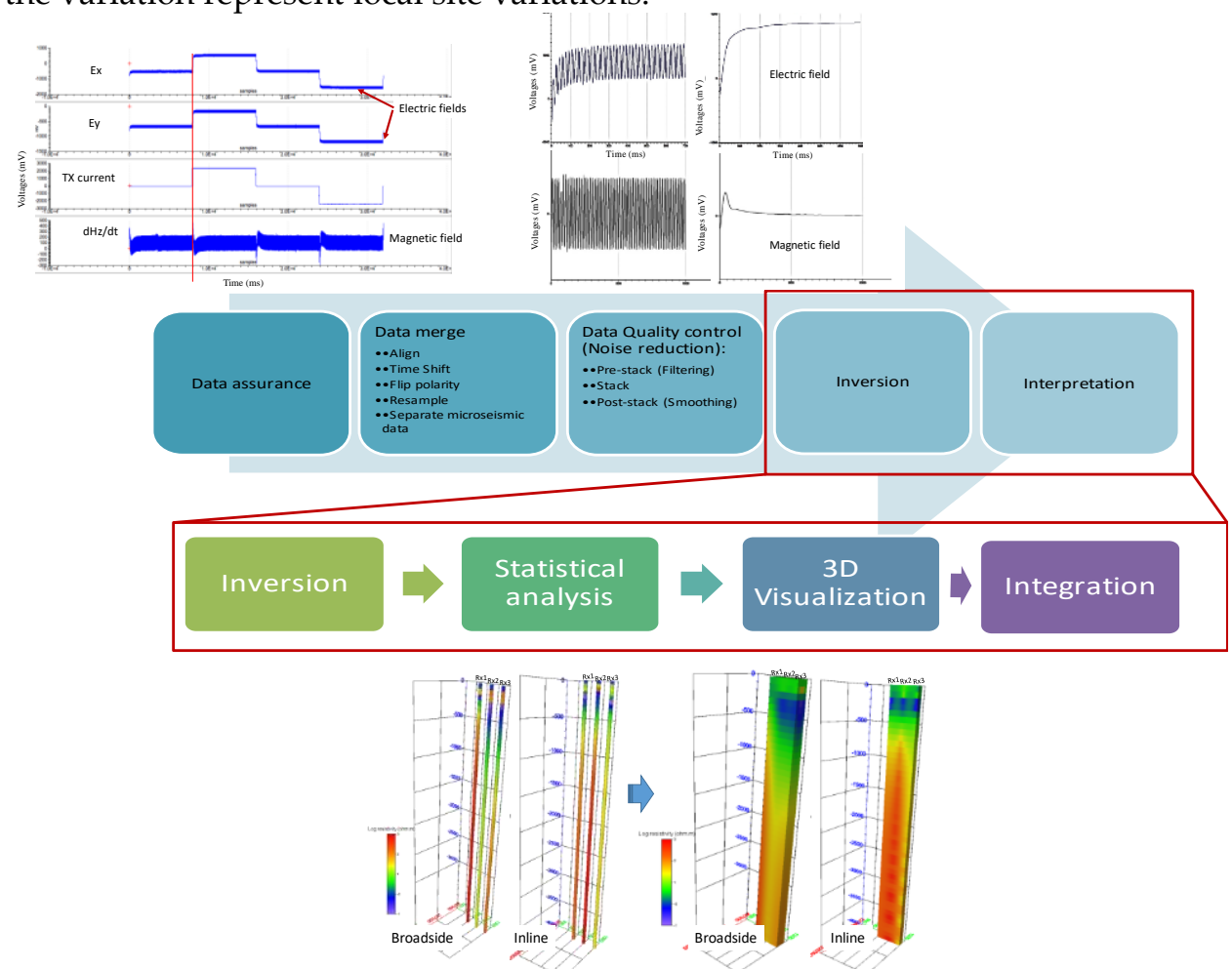

Figure 23: Processing workflow for CSEM. At the top of the workflow are a section of the raw data. To its right are the robust stacked electric (top) and magnetic fields 
(bottom). The one on the right is after filtering period noise (mostly cultural). At the bottom of the figure, we have the inversion results from a smoothness constrained inversion with its $3 \mathrm{D}$ rendering on its left.

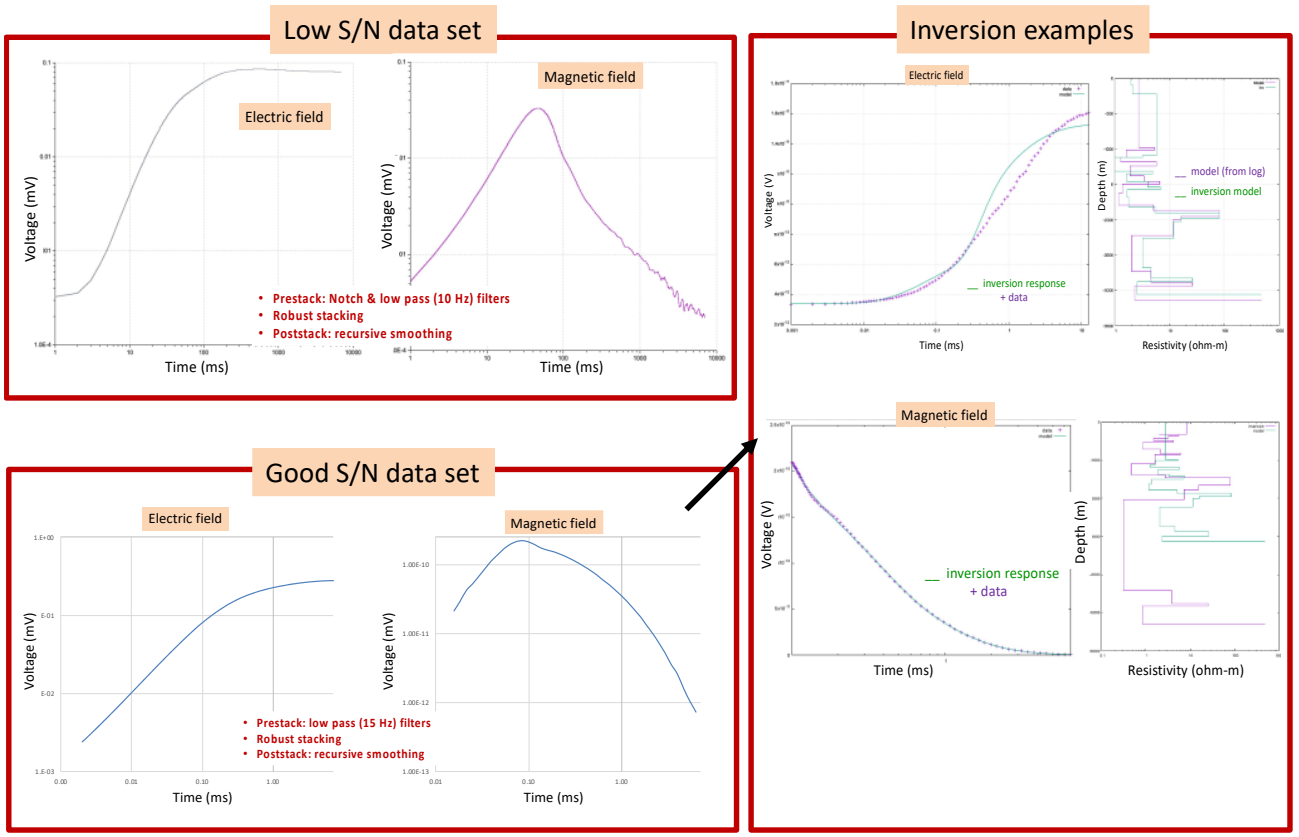

Figure 24. Example of monitoring data for electric and magnetic fields. On the left we have low signal-to-noise data (top) and good signal to noise data at the bottom. The data is displayed logarithmic to show the smoothness at later times better. On the right are the initial inversion results for the better data. The inversion model on the right and the anisotropic starting model (log derived) are displayed. The small variation between the model means that the data is very close to the logs.

When designing an EM system, we must translate the requirements to specifications and include geophysical operational aspects. We attempt to describe in this section our solution in a generic fashion to avoid commercial bias as much as we can. We can learn a lot from the seismic hardware industry since they have gone through many generations of equipment evolution. Given that increased use of EM geophysics will only happen if the majority of geophysicist (namely seismologists) embrace the methods and operation that seem to be a safe approach. In addition, most cost in field operation is in logistics, HSE and workflow, which leads us to take the approach to standardize our view with seismic equipment in mind. Thus, field operations are a significant driver in the specifications. Table 2 shows a summary of specifications of an EM receiver derived from above requirements, the technical discussion, and operational concerns. We derive our operations specifications on the survey layouts in Figure 3 and follow comment on each of the system components shown in Figures 4 and 7. Here, we described each component and its design starting with the common unit, the receiver, data acquisition system or node, then the sensors, then the transmitter, and finally the integrating software.

Table 2. Summary of basic specification for an EM receiver system. Note different manufacturers have slightly different specifications and a comprise is taken here.

\begin{tabular}{cccc}
\hline$\#$ & Item & Specifications & Comments \\
\hline & Watertight, operated closed & \\
1 & Field equipment enclosure & $\begin{array}{c}\text { Not too small to be easily lost } \\
\text { Not too heavy }\end{array}$ & IP67 standard \\
\hline 2 & Power usage - receiver & $<5 \mathrm{~W}$ & Powering all sensors \\
& plus external battery operation & included \\
\hline 3 & Bcquisition unit: & Broadband: DC $-20 \mathrm{kHz}$ & For MT \& CSEM \\
\hline
\end{tabular}




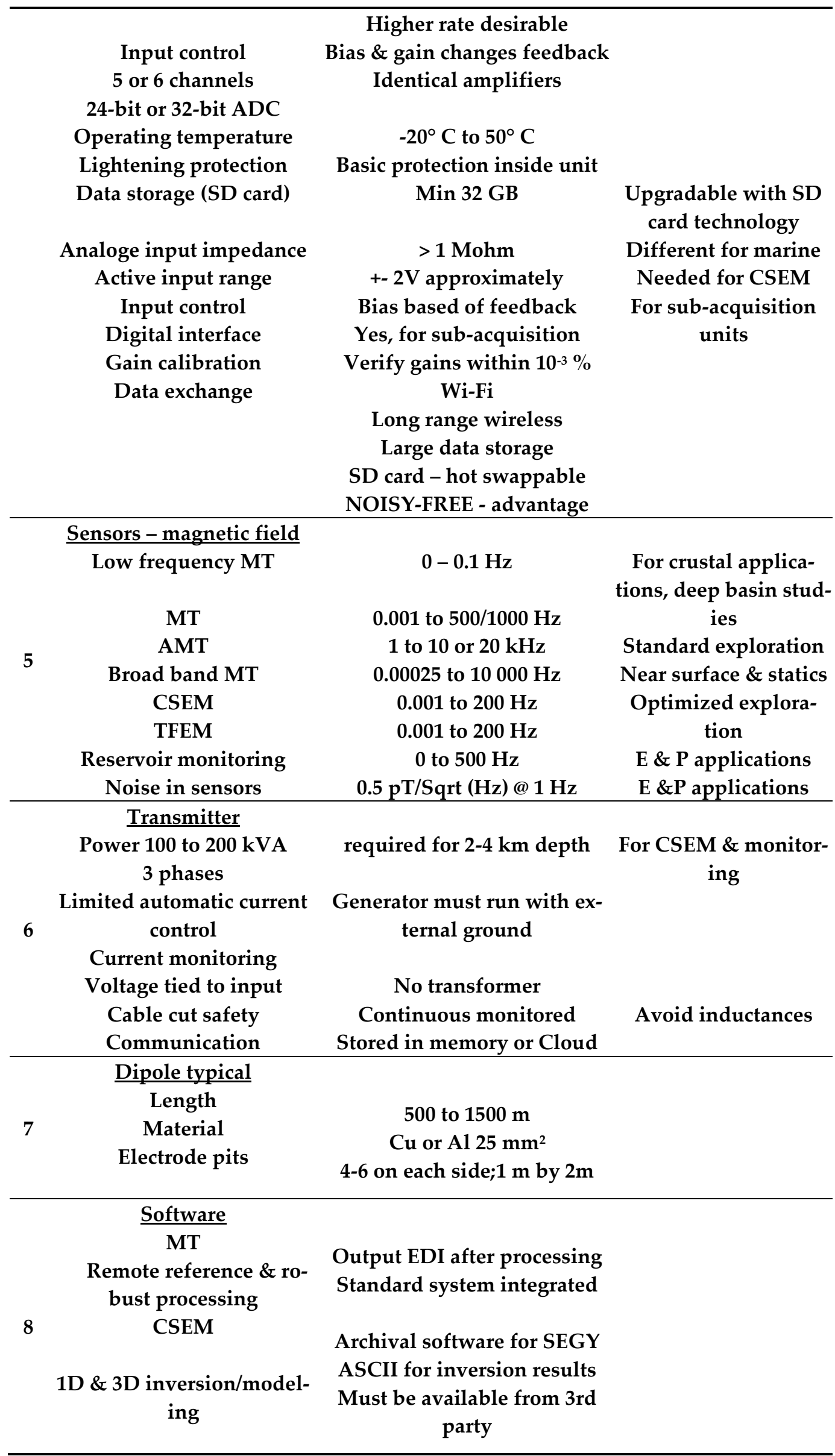


Field operations requires a system that can continuously run for an extended period. Power usage should be low but since EM requires special amplifiers it will never be as low as seismic nodes. Operations should give design priorities as this is where the operational cost saving is. However, transparency of data flow and all setting and calibrations in the system is a must and raw time series must be saved. For marine systems a good overview can be found in [82]. Except for the input resistance of the amplifiers, marine and land system are very similar with land having more flexibility in methodology, but also a much larger potential for operational errors

The acquisition node usually has additional processing capabilities and has multiple functionalities. It has evolved a long way from standard a data logger. To best match an acquisition system to the specific EM frequency band, method, and noise influence (like very low frequency magnetic field drift; self-potential; Earthquake EM signatures etc.) survey objectives must be added to the specifications. Recent developments in high resolution analogue to digital converters make analogue filters almost completely obsolete. Today, it is possible to sample the input signals with the same accuracy as before, but without removing beforehand unwanted signals like power line harmonics. Unlike analogue filters, digital filters can be optimized to be effective in time and frequency domain at the same time. While magnetic field amplifiers are critical to recover useful signal, electrical field amplifier require the same care to avoid potential thermal effects that can cause drift when exposed to sunlight or different temperatures. The difference to seismic amplifiers lies in the much broader bandwidth as modern EM system acquire over six decades of frequencies, whereas seismic can live with one. Modern systems design requires adaptation to different EM methods and tailoring the technique to the best target acquisition. For CSEM applications the correction may have to be done in a short dead-time just before the CSEM signal is being generated to achieve very accurate signal. This is true for time lapse applications where measurement accuracy and stability for a long period is paramount. The question of 24 versus 32-bit acquisition is not a real issue (our dynamic rage is limited by signal and noise) and slowly all 24-bit ADC will be replaced by 32-bit if they can acquire sufficiently high frequencies.

The sensor side is one of the most important sides and operational care/calibration should be taken into consideration. For the electric field measurement, the contact/grounding resistance should be kept stable, which can be easily achieved via routine monitoring. Manual monitoring by the operator is preferable during the survey setup to ensure automatic signal correction. The human interaction guarantees it is integrated into survey's workflow and always observed. Capacitive electrodes are limited at the lower end of the frequency band and cause unpredictable and hard to see signal distortions like using a low-cut filter in the amplifier (good capacitive electrodes work for the AMT range though). Carefully built electrodes are usually good for several years, though as careful geophysicist you should replace them at least annually as they can become the source of noise. The electric field is one of the most important measurements and the sensors are relatively inexpensive. Since most operational errors come from here, we emphasize that there always must be focus on the electric field sensors. Many magnetic field sensors exist, but only induction coil magnetometers and fluxgate magnetic field sensors have maintained their place in the market. SQUID (Super-conducting Quantum Interference Device) were very popular in the 1980s [83] because they can be up to 1 million times more sensitive than coils, but they are very difficult to handle in operations and are at least 10 times more expensive thus do not allows the deployment of several tens or hundreds of these sensors. That leaves them for research purposes until the cost come down and operations get improved. Inductions coil magnetometers are sensors of choice for MT while fluxgate magnetometers are used for lower frequencies. A lightly outdated summary of the standard commercially available induction coils can be found in [84]. Induction coils have magnetic core made of material with high magnetic permittivity (permalloys or laminated ferrite). Therefore, they may affect each other and should be kept at least 1 to $2 \mathrm{~m}$ apart during calibration and recording. The sensors discussion above covers the basic EM sensors, individual companies have now optimized broadband sensors for a variety of 
applications. While they are slightly noisier than the historic BMT/AMT sensors, their cost savings in asset purchase (about $60 \%$ of their equivalent BMT/AMT set) and operational cost out-weight this in most commercial cases (see Figure 5 for sensor noise density curves). Fluxgate [85] use an active saturation of a magnetic core and a pickup coil that measures the modulated signal by the magnetic field, which is demodulated to obtain the actual field values. Thus, they work in a balance point like all commercial induction logging tools (that measure while moving in the borehole) and are less sensitive to physical movement (seismic and wind noise) but more sensors to moving metal. For our purposes they work better in windy terrain or for airborne applications if the frequency limitation is not a problem. We use them for land, marine MT, and borehole applications and put digitization near the sensors. Since the bandwidth for seismic acquisition is so small, almost any EM acquisition system can acquire data with seismic sensors if sampling rate and filters can be set in a similar fashion. In the future, as noise control/compensation improves, we foresee the digitization to get integrated into the sensors to further reduce cost and simplify operations. Cables and battery operations should follow seismic industry standard to avoid issues with livestock and weight/cycle time, respectively. Here, we should strictly follow the seismic industry that has gone thorough many optimization cycles. Saving money in cables or connectors often results in operation time lost.

For CSEM measurements with a target depth of 2-4 km a high-power grounded dipole transmitter is required. This adds significant operational difficulties and safety concerns $[55,57]$. Using a grounded dipole is the only way to generate the vertical current flow that is needed to see thin resistive layers that are typical for hydrocarbon reservoirs $[26,28,29]$. Since we acquire many data sets for each transmitter signal, it is important that transmitter parameters are stable.

From geophysical side the only custom component we need to add to the transmitter is the switchbox responsible for making the current waveform, monitoring transmitter safety, and recording operational parameters. Over the past 35 years many special-purpose high power transmitters were built, but they never made it into commercial application. Today's high-power solid-state switches changes this, so that they allow us to use almost any input three phase generator to supply the transmitter power. Since we are grounding the transmitter via the dipole, its power should be separated from the control power and receiver site installation power to avoid ground loops and automatic ground fault shut offs. Safety on the transmitter side is of highest concern, and safety devices to turn off the transmitter due to transmitter dipole vandalism, generator diesel supply issues, and control electric and operations related issues should be included.

The weak link in transmitter stability are the electrode plants. Larger surface area of the individual electrodes and keeping them wet will provide stable groundings. You want to avoid making galvanic cells and heat up the water too much with the transmitter current as it will burn out the electrodes which causes operational interrupts. The best way is to dig many (like 6 to 10) pits on each electrode end and to control the dipole resistance with the transmitter wire thickness. Then the switchbox can handle the current control, as there is no more dynamic weak link in the circuit.

Data formats are always an issue, and since EM applications grow so slowly, EM standards never quite reach common use. Thus, we recommend using only adopted standards like EDI format (Society of Exploration Geophysicist's standard for MT data) or use conversion routine to SEGY as part of the workflow for archiving and backup. Since many scientific groups are working on other standards, this allows converting the data to whatever format you need, if you have a raw data reader.

Because of the advances in cell phone/computer technology, there has been recent emphasis on internet control of the EM node. Unlike with seismic, magnetic field sensors are extremely sensitive and often record earthquakes quite far away. Since the sensors include magnetic material, they interact with each other and pick up cell phone and RF noise. While several manufacturers advertise using web control, they recommend turning of wireless access during recording. This can be overcome if you take the wireless transmission away from the analogue electronics either wired or low noise Wi-Fi. Figure 25 
shows an example of doing this. Shown are frequency spectra in a city environment with relatively high background noise. On the left the web-access box is away from all analog parts, and data transmission (bottom) gives the same spectrum as disabled. The spectra are almost the same, and in this arrangement the unit can continuously communicate with the internet while recording. On the right, the data transmission to the internet happens near the analog sensors, cables, or acquisition unit. For MT measurements this is critical, while for CSEM it is less critical if the signal is strong.

The biggest advantage for real time streaming is the access to Cloud based applications and the use of artificial intelligence/deep learning to achieve more simplicity and optimization. This will take it to the real breakthrough of significantly enlarging the application envelope [43].

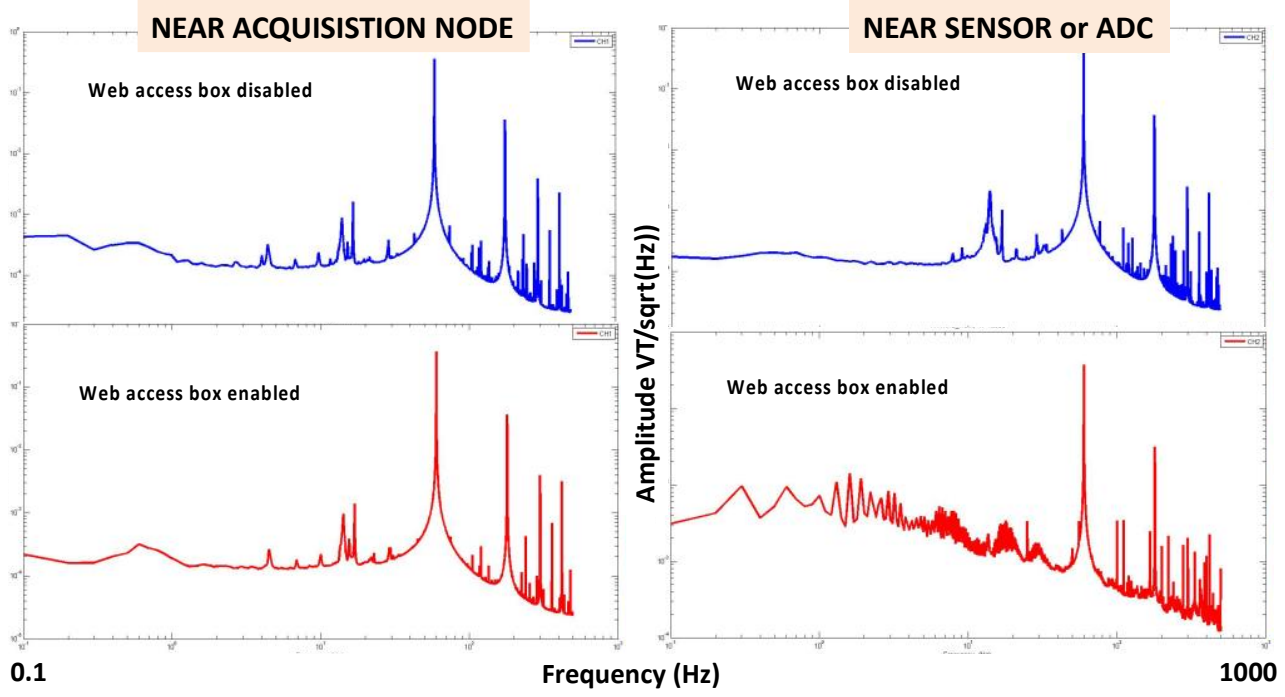

Figure 25. Frequency responses showing the influence of web data transmission when the transmission unit is near the sensor or analog part of the electronics. On the left, we have the web-access unit just outside the node away from sensors and analog electronics. On the right, the web-access unit is near the sensors and the analog cable.

\section{Summary and Outlook}

Given the success of seismic acquisition, we have described an EM system architecture, methodology and processing that allows the combination of seismic and EM acquisition by the same crew thus avoiding the duplication of the largest cost components (operations and logistics). Since the 1980s when the first seismic acquisition systems were adapted for EM acquisition, we have come a long way and today a seismic crew can easily acquire EM data and processing algorithms are similar flexible as they are in seismic data processing. Starting around the early 2000, EM has shown significant value to the exploration and new borehole tools have come online to integrate surface and borehole data. Parallel to that the entire technology spectrum has been improved. We use 3D modeling and new methodologies to illustrate with case histories how EM can be used for reservoir monitoring. Here, the combination of EM methods and seismic is ideal as seismic defines accurately the boundaries of the reservoir and EM the fluid changes.

We combine hardware design with operational, processing, and interpretation requirements to obtained optimized specifications. These and the integration of 3D modeling from start to finish of a survey allowed us to get the successful results plus we can define the next generation of hardware which includes a borehole receiver. In all case histories the surface measurements match the log and when they were where drilled, the wells were successful. In the monitoring applications the data and 3D modeling is consistent with logs and fluid injection history.

To utilize the methodology in practical geophysical context for reservoir monitoring, we need to review instrumentation from basic sensors selection, transmitter and receiver, 
acquisition, field operations, data processing and interpretation. Except for interpretation - which is more subjective- we address these technology components to understand where the errors and uncertainties occur.

To have similar good quality images as seismic routine delivers and to ease the integration in interpretation, we must get more comparable data density. This requires new system architecture and careful understanding of the differences between hardware and data handling to further support cost reduction and improve operational efficiency. Since EM loses sensitivity with depth faster than seismic, it is necessary to add borehole calibration measurements in production scenarios. For that, the system including high-power transmitter, three-component electric and magnetic receivers and $3 \mathrm{C}$ geophones has been developed, tested in field and is ready for application. The next big breakthrough is expected when Cloud base data delivery and artificial intelligence-based processing and interpretation are being rolled out commercially.

We illustrated the benefits of focusing methods by using FSEM and shallow borehole measurements, since they are the most sensitive to vertical currents significantly affected by a resistive oil reservoir. Methods like this will be required to bring the error of processing and interpretation to a comparable error level of the measurements. These measurements can be utilized in a variety of ways adopted to field operations. All field measurements and methodologies as well as hardware design are carefully verified by 3D modeling and where possible field test.

While fluid imaging in general is very useful and directional sensitivity can significantly improve the insight in the reservoir structure. The biggest value will come from integrating this to the energy transition when we direct the technology to geothermal reservoirs and apply it to carbon storage applications. Combining carbon capture with EOR will not only improve the recovery factor thus reducing the carbon footprint per barrel produced but also use $\mathrm{CO}_{2}$ for production enhancement while string it underground. Initial field trials for this are well underway.

Supplementary Materials: The following are available online at www.mdpi.com/xxx/s1, Figure S1: title, Table S1: title, Video S1: title.

Author Contributions: Lead author, concepts, and technology details, K.S.; 3D modeling, S.D.; software and MT advice, M.S.; sensors and geothermal field test, TH; CSEM processing and overall support, AYP. All authors have read and agreed to the published version of the manuscript.

Funding: This research received no external funding. It is part of our corporate background technology used in various projects

Data Availability Statement: Data supporting this can be found at www.kmstechnologies.com

Acknowledgments: We highly appreciate the support and technical expertise of the dedicated personnel at KMS Technologies. We appreciative for support from our clients throughout these years including but not limited to Geokinetics, Ormat, PTTEP, and various customers that purchased the system.We acknowledge the support and contribution of X. Xu, Y. Martinez, T. Tasci, and H. Hinajosa during the more recent development development. KMS Technologies gave us permission to publish this material. A thank you to everyone.

Conflicts of Interest: The authors declare no conflict of interest.

\section{References}

1. Strack, K.M., Future Directions of Electromagnetic Methods for Hydrocarbon Applications. Surveys in Geophysics, 2014, 35, 157-177, DOI: 10.1007/s10712-013-9237-z.

2. Passalacqua, H., and Strack, K., Reducing carbon footprint by geophysical monitoring of EOR processes. Technical program expanded abstract 2020, Soc. Expl. Gephys., 2020, 3384-3398, doi: 10.1190/segam2020-3424907.1.

3. Vozoff, K., The magnetotelluric method in the exploration of sedimentary basins. Geophysics, 1972, 37, 98-141, doi: $10.1190 / 1.1444178$ 
4. Vozoff, K., The magnetotelluric method. in Nabighian, M. N., Ed., Electromagnetic methods in applied geophysics. Society of Exploration Geophysics, 1991, 2B, 641-711, doi: 10.1190/1.1444178.

5. Nekut, A.G., and Spies, B.R, Petroleum exploration using controlled-source electromagnetic methods. Proc. IEEE, 1989, 77, 338-362, doi: 10.1109/5.18630.

6. Spies, B.R., and Frischknecht, F.C., Electromagnetic soundings. in Nabighian, MN, Electromagnetic Methods in Applied Geophysics: Volume 2, Application, Parts A and B. Society of Exploration Geophysicists, 1991, doi: 10.1190/1.9781560802686.ch5.

7. Nabighian, M.N., and Macnae, J.C., Electrical and EM methods. 1980-2005. The Leading Edge, 2005, 24, 42-45, doi: 10.1190/1.2112391.

8. Sheard, S., Ritchie, T., Christopherson, K., and Brand, E., Mining, Environmental, Petroleum, and Engineering Industry Applications of Electromagnetic Techniques in Geophysics. Surveys in Geophysics, 2005, 26, 653-669, doi: 10.1007/s10712-005-1760-0.

9. Constable, S. and Srnka, L.J., An introduction to marine controlled-source electromagnetic methods for hydrocarbon exploration. Geophysics, 2007, 72, WA3-WA12, doi: 10.1190/1.2432483.

10. Constable, S., Ten years of marine CSEM for hydrocarbon exploration. Geophysics. 2010, 75, 75A67-75A81, doi: 10.1190/1.3483451.

11. Streich, R., Controlled-Source Electromagnetic Approaches for Hydrocarbon Exploration and Monitoring on Land. Surveys in Geophysics, 37, 47-80, 2016, doi: 10.1007/s10712-015-9336-0.

12. Spies, B.R., Recent developments in the use of surface electrical methods for oil and gas exploration in the Soviet Union. Geophysics, 1983, 48, 1102-1112, doi: 10.1190/1.1441532.

13. Henke, C. H., Krieger, M., Strack, K., and Zerilli, A., Subsalt imaging in Northern Germany using multi-physics. Interpretation, 2020, 8, SQ14-SQ24, doi: 10.1190/INT-2020-0026.1.

14. Colombo, D., McNeice, G., Curiel, E.S., and Fox, A., Full tensor CSEM and MT for subsalt structural imaging in the Red Sea: Implications for seismic and electromagnetic integration. The Leading Edge, 2013, 32, 436-449, doi: 10.1190/tle32040436.1.

15. Colombo, D., Keho, T., and McNeice, G., Integrated seismic-electromagnetic workflow for sub-basalt exploration in northwest Saudi Arabia. The Leading Edge, 2012, 31, 42-52, doi: 10.1190/1.3679327.

16. Carlson, M.R., An Analysis of the Caprock Failure at Joslyn. SPE Heavy Oil Conference in Calgary, Alberta, 1214th June 2012, SPE-156962-PP, doi: 10.2118/156962-MS

17. Ceia, M., Carrasquilla, A., Sato, H., and Lima, O., Long Offset Transient Electromagnetic (LOTEM) for monitoring fluid injection in petroleum reservoirs - Preliminary results of Fazenda Alvorada Field (Brazil). 10th International Congress of The Brazilian Geophysical Society, 2007, doi: 10.1190/sbgf2007-013

18. Colombo, D., Dasgupta, S., Strack, K.M., and Yu, G., Results of Feasibility Study of Surface-to-Borehole Time-Domain CSEM for Water-Oil Fluid Substitution in Ghawar Field. Saudi Arabia. 2010, doi: 10.3997/2214-4609pdb.248.435.

19. Strack, K.M., and Aziz, A.A., Full Field Array ElectroMagnetics for hydrocarbon reservoir exploration and monitoring. Geohorizons, 2013, Special Issue on Shale Gas, 18, 32-43. 10.3997/2214-4609.20148417.

20. Kumar, D., and Hoversten, G.M., Geophysical model response in a shale gas. Geohorizons, 2012, 17, 31-37, 2012. 
21. Passalacqua, H., Davydycheva, S., and Strack, K., Feasibility of multi-physics reservoir monitoring for Heavy Oil. Heavy Oil Conference Kuwait, 2018, SPE-193690-MS, 10.2118/193690-MS

22. He, Z., Liu, X., Qiu, W., and Zhou, H., Mapping reservoir boundary by borehole-surface TFEM: Two case studies. The Leading Edge, 2005, 24, 896-900, doi: 10.1190/1.2056379.

23. He, Z., Hu, W., and Dong, W., Petroleum Electromagnetic Prospecting Advances and Case Studies in China. Surveys in Geophysics, 2010, 31, 207-224, doi: 10.1007/s10712-009-9093-z.

24. Tietze, K., Ritter, O., and Veeken, P., Controlled-source electromagnetic monitoring of reservoir oil saturation using a novel borehole-to-surface configuration. Geophysical Prospecting, 2015, 63, 1468-1490, doi:

https://doi.org/10.1111/1365-2478.12322.

25. Kalscheuer, T., Juhojuntti, N., and Vaittinen, K., Two-Dimensional Magnetotelluric Modelling of Ore Deposits: Improvements in Model Constraints by Inclusion of Borehole Measurements. Surveys in Geophysics, 2018, 39, 467507, doi: 10.1007/s10712-017-9454-y.

26. Strack, K.-M., Lebrocq, K., Moss, D. C., Petry, H., Vozoff, K., and Wolfgram, P. A., Case histories of long-offset transient electromagnetics (LOTEM) in hydrocarbon exploration. First Break, 1989, 7, 467-477, DOI: 10.3997/13652397.1989027.

27. Thiel, S., Electromagnetic Monitoring of Hydraulic Fracturing: Relationship to Permeability, Seismicity, and Stress. Surveys in Geophysics, 2017, 38, 1133-1169, doi: 10.1007/s10712-017-9426-2.

28. Passalacqua, H., Electromagnetic Fields due to a Thin Resistive Layer. Geophysical Prospecting, 1983, 31, 945-976, doi: 10.1111/j.1365-2478.1983.tb01099.x.

29. Eadie, T., Detection of hydrocarbon accumulations by surface electrical methods - a feasibility study. Research in Applied Geophysics, 1981,15, University of Toronto, https://hdl.handle.net/1807/69190.

30. Eidesmo T., Ellingsrud, S., MacGregor, L. M., Constable, S., Sinha, M. C., Johansen, S., Kong, F. N. and Westerdahl, H., Sea Bed Logging (SBL), a new method for remote and direct identification of hydrocarbon filled layers in deep water areas. First Break, 2002, 20, 144-152, doi: 10.1046/j.1365-2397.2002.00264.x.

31. Barber, T., Anderson, B., Abubakar, A., Broussard, T., Chen, K-C., Davydycheva, S., Druskin, V., Habashy, T.M., Homan, D.M., Minerbo, G., Rosthal ,R., Schlein, R. and Wang, H., Determining formation resistivity anisotropy in the presence of invasion. Society of Petroleum Engineers, 2004, Annual Technical Conference, Paper 90526, doi: 10.2118/90526-MS.

32. Yu, L., Fanini, O., Kriegshaeuser, B., Koelman, J.M.V., and Popta, J., Enhanced evaluation of low-resistivity reservoirs using multi-component induction log data. Petrophysics,2001, 42, 611-623.

33. Keller, G.V., and Frischknecht, F.C., Electrical Methods in Geophysical Prospecting. Elsevier, 1966, ISBN: 9780080115252.

34. Fanini, O., Haines, H., Hunziker, J., Maurer, H.-M., R. Siegfried II, and Strack, K.-M., 1999, A Major Technology Breakthrough - Cased-Hole Resistivity Tool. In-depth 5, 12-25.

35. Strack, K.-M., Tabarovsky, L.A., Beard, D.B., and van der Horst, M., Determining electrical conductivity of a laminated earth formation using induction logging. US patent 6,147,496, 2000.

36. Strack K.-M., Combined surface and wellbore electromagnetic measurement system and method for determining formation fluid properties. US patent 6,739,165 B1, 2004.

37. Davydycheva, S., and Rykhlinski, N., Focused-source electromagnetic survey versus standard CSEM: 3D modeling in complex geometries. Geophysics, 2011, 76, doi: 10.1190/1.3511353. 
38. Davydycheva, S., Kaminsky, A., Rykhlinski, N., and Yakovlev, A., A large-scale field study in eastern Siberia using novel time-domain electromagnetic technology. Interpretation, 2015, 3, T109-T120, doi: 10.1190/INT2013-0165.1.

39. Strack, K., and Davydycheva, S, Using electromagnetics to map lateral fluid variations in carbonates in SE Asia. in Purenovic, J., ed., New approaches in engineering research vol. 2. BP International, 2021, doi: 10.9734/bpi/naer/v2/8202D

40. Strack, K.-M., and Vozoff, K., Integrating long-offset transient electromagnetics (LOTEM) with seismics in an exploration environment. Geophysical Prospecting, 1996, 44, 99-101, doi: 10.1111/j.1365-2478.1996.tb00188.x.

41. Strack, K., and Pandey, P.B., Exploration with controlled-source electromagnetic under basalt cover in India. The Leading Edge, 2007, 26, 268-27, DOI: 10.1190/1.2715056.

42. Strack K.-M. 2004. Combined surface and wellbore electromagnetic measurement system and method for determining formation fluid properties. US patent 6,739,165 B1.

43. Strack, K., Davydycheva, S., Passalacqua, H., Smirnov, M.Y., and Xu, X. Using Cloud-Based Array Electromagnetics on the Path to Zero Carbon Footprint during the Energy Transition. Preprints, 2021, 2021080009, doi: 10.20944/preprints202108.0009.v1.

44. Jiang, J., Aziz, A.A., Liu, Y., and Strack. K.M., 2015, Geophysical acquisition system. US 9,057,801.

45. Torres-Verdín, C., and Bostick, F.X., Principles of spatial surface electric field filtering in magnetotellurics: Electromagnetic array profiling (EMAP). Geophysics, 1992, 57, 603-622, doi: 10.1190/1.1443273.

46. Vozoff, K., The magnetotelluric method in the exploration of sedimentary basins. Geophysics, 1972, 37, 98-141, doi: 10.1190/1.1440255.

47. Vozoff, K., Magnetotellurics: Principles and practice. Proc. Indian Acad. Sci. - Earth Planet. Sci., 1990, 99,441471, doi: 10.1007/BF02840313.

48. Simpson, F., and Bahr, K., Practical Magnetotellurics. Cambridge: Cambridge University Press, 2005. 10.1017/CBO9780511614095.

49. Chave, A.D., and Jones, A.G., The Magnetotelluric Method, Cambridge University Press, 2012, 10.1017/CBO9781139020138.

50. Cumming, W., and Mackie, R., Resistivity imaging of geothermal resource using 1D, 2D, and 3D MT inversion and TDEM static shift correction illustrated by a Glass mountain case history. Proc. World Geotherm. Congr., 2010.

51. Amatyakul, P., Rung-Arunwan, T., and Siripunvaraporn, W., A pilot magnetotelluric survey for geothermal exploration in Mae Chan region. Northern Thailand. Geothermics, 2015, 55, 31-38, doi 10.1016/j.geothermics.2015.01.009

52. He, Z., Hu, Z., Gao, Y., He, L., Meng, C. and Yang, L., Field test of monitoring gas reservoir development using time-lapse continuous electromagnetic profile method. Geophysics, 2015, 80, WA127-WA134 , doi: 10.1190/geo2014-0195.1.

53. Strack, K.M., Tabarovsky, L.A., Beard, D. R., and Van Der Horst, M. Determining electrical conductivity of laminated Erath formation using induction logging. US patent 6,147,496, 2000.

54. Keller, G.V., and Frischknecht, F.C, Electrical methods in geophysical prospecting, 1st. ed., Pergamon Press, Oxford, 1966.

55. Strack, K.M., Exploration with Deep Transient Electromagnetics. Elsevier, Amsterdam, 373 pp, 1992. 
56. Kaufman, A.A., and Keller, G.V., Frequency and Transient Sounding Methods. Elsevier, Amsterdam, 685 pp., 1984.

57. Keller, G.V., Pritchard, J.I., Jacobson, J.J., and Harthill, N., Megasource time-domain electromagnetic sounding methods. Geophysics, 1984, 9, 993-1009, doi: 10.1190/1.1441743.

58. Sheriff, R.E., Encyclopedic Dictionary of Applied Geophysics. Soc. Explor. Geophys., Tulsa, 2002,429 pp.

59. Rueter, H., and Strack, K.-M., Method of processing transient electromagnetic measurements in geophysical analysis. US patent 5,467,018.

60. Davydycheva, S., and Rykhlinski, N., Focused-source EM survey versus time-domain and frequency-domain CSEM. Geophysics, 2009, 28, doi: 10.1190/1.3192841.

61. Davydycheva, S., Method and apparatus for detecting and mapping subsurface anomalies. US patent 9,891,339.

62. Doll, H.G., The Laterolog: A new resistivity logging method with electrodes using an automatic focusing system. Journal of Petroleum Technology, 1951, 3, 305-316, doi: 10.2118/951305-G

63. Davydycheva, S., and Druskin, V., Staggered Grid for Maxwell's Equations in 3-D Anisotropic Media. in Oristaglio, M.L., and Spies, B.R., eds., Three-Dimensional Electromagnetics. Society of Exploration Geophysicists, Tulsa, 1999, doi: 10.1190/1.9781560802150.ch9.

64. https://kmstechnologies.com/Files/Flyer\%20for\%20website/CSEMulatorDataSheet_1.2.pdf

65. Paembonan, A.Y., Arjwech, R., Davydycheva, S., Smirnov, M., and Strack, K.M., An application of LOTEM around salt dome near Houston, Texas. AIP Conference Proceedings, 2017, 1861, 30006, doi: 10.1063/1.4990893.

66. Haroon, A., Lippert, K., Mogilatov, V., and Tezkan, B., First application of the marine differential electric dipole for groundwater investigations: A case study from Bat Yam, Israel. Geophysics, 2018, 83, B59-B76, doi: 10.1190/geo2017-0162.1

67. LeFever, J.A., Horizontal drilling potential of the Middle Member Bakken. AAPG Rocky Mountain Sectional Meeting, Jackson, Wyoming, 2005, 27 pp.

68. Palish, T., Al-Tailji, W., Bartel, L., Cannan, C., and Zhang, J., Far-field proppant detection using electromagnetic methods - latest field results. SPE, 2017, Hydraulic Fracturing Technology Conf., Paper 184880-MS.

69. Tabarovsky, L.A., Cram, M.E., Tamarchenko, T.V., Strack, K.-M., and Zinger, B.S., Through-Casing Resistivity (TCR) Physics, Resolution and 3-D Effects. Presented at SPWLA, Thirty-Fifth Annual Logging Symposium, Tabarovsky, L.A., Cram, M.E., Tamarchenko, T.V., Strack, K.-M., and Zinger, B.S., 1994, Through-Casing Resistivity (TCR) Physics, Resolution and 3-D Effects: Presented at SPWLA, Thirty-Fifth Annual Logging Symposium, Paper TT. , Paper TT.

70. Zhou Q, Julander D, Penley L., Experiences with cased hole resistivity logging for reservoir monitoring. SPWLA 43rd Annual Logging Symposium. Oiso, Japan, 2002, paper X.

71. Strack, K.-M., Hanstein, T. H., and Eilenz, H. N., LOTEM data processing for areas with high cultural noise levels. Physics of the Earth and Planetary Interior, 1989, 53, 261-269, doi: 10.1016/0031-9201(89)90010-1.

72. Ziolkowski, A., Hobbs, B., and Wright, D., Multitransient electromagnetic demonstration survey in France. Geophysics, 2007, 72, F197-F209, doi: 10.1190/1.2735802.

73. Mörbe, W., Yogeshwar, P., Tezkan, B., and Hanstein, T., Deep exploration using long-offset transient electromagnetics: interpretation of field data in time and frequency domain. Geophys. Prosp., 2020, 68, 1980-1998, doi: $10.1111 / 1365-2478.12957$ 
74. Smirnov, M., Magnetotelluric data processing with a robust statistical procedure having a high breakdown point. Geophysical Journal International, 2003, 152, 1, DOI: 1 10.1046/J.1365-246X.2003.01733.X

75. Siegel, A.F., Robust regression using repeated medians. Biometrika, 1982, 69, 242-244, doi: 10.1093/biomet/69.1.242

76. Buehnemann, J., Henke, Ch., Krieger, M., Mueller, Ch., Strack, K.-M., and Zerilli, A., Bringing complex structure into focus - A novel integrated approach. Soc. Expl. Geophys., Expanded Abstracts, 2002, 446-449, doi: $10.1190 / 1.1817278$

77. Egbert, G.D., Robust Multiple Station Magnetotelluric Data Processing. Geophys. J. Int., 1997, 130, 475-496, doi: 10.1111/j.1365-246X.1997.tb05663.x

78. Larsen, J. C., Low frequency (0.1-6.0 cpd) electromagnetic study of deep mantle electrical conductivity beneath the Hawaiian Islands. Geophys. J. R. Astr. Soc., 1975, 43, 17-46, doi:

79. Bahr, K., Geological noise in magnetotelluric data: a classification of distortion types. Phys. Earth Planet. Inter., 1991, 66, 24-38, doi: 10.1111/j.1365-246X.1997.tb01859.x

80. Pankratov, O.V., and Geraskin, A.I., On processing of Controlled Source Electromagnetic (CSEM) Data. Geologica Acta, 8, 2010, 31-49 doi: 10.1344/105.000001514

81. Spies, B. R., Depth of investigation in electromagnetic sounding methods. Geophysics, 1989 54, 872-888, doi: 10.1190/1.1442716.

82. Constable, S., Review paper: Instrumentation for marine magnetotelluric and controlled source electromagnetic sounding. Geophys. Prosp., 2013, 61, 505-532, doi: 10.1111/j.1365-2478.2012.01117.x

83. Weinstock, H., and Overton, W.C. Jr., SQUID applications in geophysics. Society of Exploration Geophysicists., Tulsa, 1981.

84. Poliatov, S.V. Reznikov, B.I., Shchennikov, A.V., Kopytenko, E.A. and Samsonov, B.V., The range of inductioncoil magnetic field sensors for geophysical exploration. Seismic instruments, 2014, 53, 1, 1018.

85. Ripka, P., Magnetic Sensors and Magnetometers, Artech House, 2001. 